\title{
Stream macroalgal flora from Parnaíba River Basin, Brazil: reducing Wallacean shortfall
}

\author{
Marina Ramos Aurichio ${ }^{I}$, Richard Wilander Lambrecht ${ }^{2}$ \& Cleto Kaveski Peres $^{1 * \mathbb{B}}$ \\ ${ }^{1}$ Universidade Federal da Integração Latino-Americana, Instituto Latino-Americano de Ciências da Vida e da \\ Natureza, Foz do Iguaçu, PR, Brasil. \\ ${ }^{2}$ Instituto de Botânica, Núcleo de Pesquisa em Ecologia, São Paulo, SP, Brasil. \\ *Corresponding author: Cleto Kaveski Peres,e-mail: cletoperes@yahoo.com.br
}

AURICCHIO, M. R., LAMBRECHT, R. W., PERES, C. K. Stream macroalgal flora from Parnaíba River Basin, Brazil: reducing Wallacean shortfall. Biota Neotropica. 19(2): e20180685. http://dx.doi.org/10.1590/1676-0611BN-2018-0685

\begin{abstract}
The global biodiversity loss is a consensus. The biodiversity conservations shortfalls make conservation of biological diversity even more challenging. For many taxa, the knowledge about their distributions is deficient, and this is called the Wallacean shortfall. This situation is no different within algae biodiversity, especially in Brazil. There is still an enormous inequity of sample effort, as is the case of the Parnaíba River Basin (Northeast region), which had only 10 algal species in published (the lowest number of algal species reported among the main Brazilian basins). The present work had the objective of increasing the knowledge of algal flora in Brazil by conducting a taxonomic study of the stream macroalgal species of the Parnaíba River Basin. The sampling of macroalgae was carried out in 21 segments of streams from the Middle and Lower Parnaíba Basin, in the Piauí and Maranhão states. Macroalgae were manually removed and preserved in 4\% formaldehyde. Environmental characteristics of each segment were measured in order to describe the sampling sites. The taxonomic survey of the macroalgal communities resulted in the identification of 38 taxa in total, of which 32 at a specific level; three vegetative groups; two sporophytic stages of red algae and one unidentified species. Among the 38 species recorded, 37 are new records for the Parnaíba River Basin. Following the same pattern, 23 species are new records for the Brazilian Northeast region, and Microcoleus lacustris represented the first report in Brazil. Despite the fact that it remains the Brazilian basin with the lowest number of algal species documented, this study contributed to the increase of almost five times the number of species sampled in the Parnaíba River Basin (from only 10 to 47 species). These data reinforce that the differences presented in algal diversity in the Brazilian regions are more related to the sampling effort than other factors (e.g. environmental characteristics, geographic distribution, biomes, among others). In addition, this survey illustrates not only the group's lack of information in the region but also shows the importance of this type of study as a tool for expanding the knowledge about biodiversity and its conservation. Keywords: Northeast region, Piaui, Maranhão, filamentous algae, taxonomy.
\end{abstract}

\section{Flora de macroalgas de riachos da Bacia Hidrográfica do Rio Parnaíba, Brasil: reduzindo o déficit Wallaceano}

Resumo: A perda de biodiversidade é um consenso. Os déficits de conservação tornam a conservação da diversidade biológica ainda mais desafiadora. Para muitos táxons, o conhecimento sobre suas distribuições é escasso, o que é chamado de Déficit Wallaceano. Essa situação não é diferente quando tratamos da biodiversidade de algas, especialmente no Brasil. Ainda há uma enorme desigualdade no esforço amostral, como é o caso da Bacia Hidrográfica do Rio Parnaíba (região Nordeste), a qual possui apenas 10 espécies de algas (o menor número de espécies de algas dentre as principais bacias brasileiras). O presente trabalho teve como objetivo aumentar o conhecimento da flora algal no Brasil, através da realização de um estudo taxonômico das espécies de macroalgas de riachos da Bacia do Rio Parnaíba. A amostragem das macroalgas foi realizada em 21 segmentos de riachos das Bacias do Médio e Baixo Parnaíba, nos estados do Piauí e Maranhão. As macroalgas foram removidas manualmente e preservadas em formaldeído $4 \%$. Características ambientais de cada segmento foram tomadas com o intuito de descrever a área de estudo. A pesquisa taxonômica das comunidades de macroalgas resultou na identificação de 38 táxons no total, nos quais 32 em nível específico; três grupos vegetativos; dois estágios esporofíticos de algas vermelhas e uma espécie não identificada. Dentre as 38 espécies, 37 delas são novos registros para a Bacia do 
Rio Parnaíba. Seguindo o mesmo padrão, 23 espécies são novos registros para a região Nordeste, e Microcoleus lacustris representou o primeiro registro para o Brasil. Apesar do fato de permanecer como a bacia brasileira com o menor número de espécies de algas documentadas, este trabalho contribuiu para o aumento de quase cinco vezes o número de espécies amostradas na Bacia do Rio Parnaíba (de apenas 10 para 47 espécies). Estes dados reforçam que as diferenças apresentadas na diversidade algal nas regiões brasileiras estão mais relacionadas com o esforço amostral do que com outros fatores (e.g. características ambientais, distribuição geográfica, biomas, entre outros). Além disso, este trabalho ilustra não só a falta de informação do grupo na região, mas também mostra a importância deste tipo de estudo como ferramenta para expandir o conhecimento sobre a biodiversidade e conservação.

Palavras-chave: região Nordeste, Piauí, Maranhão, algas filamentosas, taxonomia.

\section{Introduction}

It is a consensus that global biodiversity is threatened and the species loss is taking place at an accelerated level (Whittaker et al. 2005). The main factors associated with this loss of biodiversity have an anthropogenic origin (Brown \& Lomolino 1998). In addition to the anthropic impacts, the Biodiversity Conservations Shortfalls make conservation of biological diversity even more difficult (Mace 2004). For many taxa, the knowledge about their global, regional, or even local distributions is insufficient, a problem that Lomolino (2004) has named the Wallacean shortfall. As a result of this lack of knowledge that many areas of the globe still remain poorly sampled, which for most taxa results in scarce basic information (Whittaker et al. 2005). In contrast, this gap could be circumvented, or at least mitigated, by investing in biodiversity inventories (Whittaker et al. 2005, Bini et al. 2006).

This scenario is no different when the algal biodiversity is approached, especially in Brazil. Despite the total number of described algae for the whole Brazilian territory (4700 species, Flora do Brasil 2020 under construction, 2018) be close to the estimated number of 5600 species in the country (Menezes et al. 2015), no doubt there's still an enormous inequality of sampling effort, with huge regions without any type of surveys. This fact not only makes it impossible for adequate management and the proposal of local conservation strategies but also creates an illusion that different regions present more species richness than others. In terms of Brazilian Hydrographic Basins (sensu Agência Nacional de Águas 2015), most surveys with this algal group cover only two basins: Southeast Atlantic (ASe) and Paraná (Pr). Both basins, according to Menezes et al. (2015), are the richest in number of species in Brazil, reaching values that exceed a thousand species. In contrast, little sampled Hydrographic Basins have a modest number of recorded species, as is the case of the Parnaíba River Basin (Pnb), which has only 10 species of algae (Menezes et al. 2015, Flora of Brazil 2020 under construction, 2018) (Figure 1). Among these species, seven are planktonic microalgae (four euglenoids, two diatoms and one cyanobacteria) and three are filamentous algae (Chara martiana, Cladophora glomerata and Hapalosiphon pumilus).

Particularly for stream macroalgae (sensu Sheath \& Cole 1992), the floristic surveys have focused basically only the Southeastern (Branco \& Necchi Júnior 1996, Necchi Júnior et al. 1997, Pereira \& Branco 2010, Almeida et al. 2011) and Southern regions of Brazil (Krupek et al. 2008, Peres et al. 2008, Branco et al. 2008, 2009, 2011). Although these efforts have contributed profoundly to the knowledge of Brazilian flora, there is a huge gap in the knowledge of the distribution of organisms throughout the territory, thus clearly contributing to the Wallacean shortfall in the group. Studies in different regions and biogeographic provinces are essential, not only providing occurrence information but also allowing an increase of reference material in herbaria. The increase of reference collections constitutes a valuable asset (Pyke and Ehrlich, 2010), also making possible future studies re-evaluating species and taxonomic positions.

In this context, the present investigation had the objective of increasing the knowledge of algal flora in Brazil by conducting a taxonomic study of the stream macroalgae species of the Parnaíba River Basin. This basin comprises a very interesting biogeographic region with the contact of three major Brazilian biomes (Cerrado, Caatinga, Amazônia, and its ecotones) and has a wide land use gradient. Moreover, as this basin presents the lowest number of algal species recorded so far (among the main Brazilian basins), it is considered as of greater relevance and a valuable contribution to the reduction of the Wallacean shortfall.

\section{Material and Methods}

The Parnaíba River Basin (Agência Nacional de Águas 2015) is located in the northeastern region of Brazil between latitudes $02^{\circ} 21^{\prime} \mathrm{S}$ and $11^{\circ} 06^{\prime}$ 'S and longitudes $47^{\circ} 21^{\prime} \mathrm{W}$ and $39^{\circ} 44^{\prime} \mathrm{W}$, encompassing the states of Ceará (4.1\% of the total area of the basin), Maranhão (19.8\%) and Piauí (75.3\%) (MMA 2006). The region is an ecotone between the Cerrado and Caatinga biomes, also influenced by Amazonian and coastal vegetation (MMA 2006). It presents a well defined seasonal water regime (rainy and dry period) with several streams being intermittent (MMA 2006). Most of the selected streams are placed in areas disturbed by the extensive farming use, typical of this region. Nevertheless, some sampled streams are inserted in low disturbed landscapes while others are inserted in very disturbed areas with an urban influence.

The sampling of macroalgae was carried out in 21 segments of streams belonging to Parnaíba River Basin, in the Piauí and Maranhão states (Figure 2, ICMBio License number 58717-3), during a period of drought and after a period of regular precipitation. The selected segments are 1 st to 3 rd stream order and comprised microbasins in different parts of the Middle and Lower Parnaíba River Basin. The collection and preservation of the algal samples followed the standard procedures described by Necchi Júnior et al. (1991). Each sampling unit consisted of a stream segment of 10 meters long. Using a transparent underwater viewer it was possible to observe and remove macroalgae, which were preserved in $4 \%$ formaldehyde. Environmental characteristics were taken in field, such as water temperature (Temp.) using a thermometer $\mathrm{Akso}^{\mathrm{TM}}, \mathrm{pH}$ using a $\mathrm{pHmeter} \mathrm{Akso}^{\mathrm{TM}}$, conductivity (Cond.) using a 


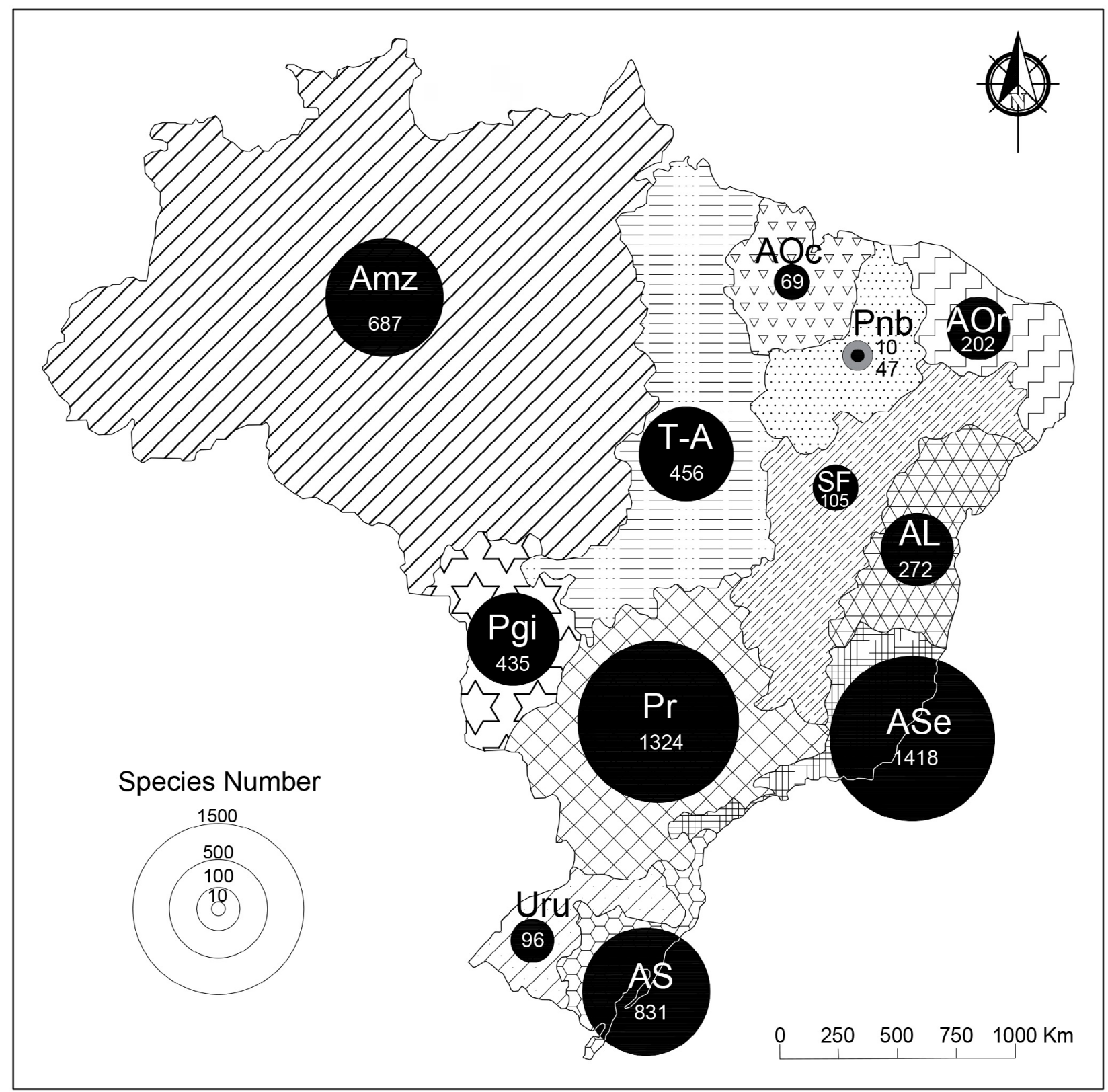

Figure 1. Species richness of epicontinental algae and cyanobacteria for each Brazilian hydrographic regions, with emphasis on the increase of species in the Parnaíba Basin. (Amz - Amazon, AL - East Atlantic, AOc - Occidental Northeast Atlantic, AOr - Oriental Northeast Atlantic, ASe - Southeast Atlantic, AS - South Atlantic, Pgi - Paraguay, Pr - Paraná, Pnb - Parnaíba, SF - São Francisco, T-A - Tocantins-Araguaia, and Uru - Uruguay).

conductivimeter $\mathrm{Akso}^{\mathrm{TM}}$, predominant substrate type (Subst.) by visual observation, according Gordon et al. (1992) classification, average current velocity (Vel.) using the floating object method, average width and depth of each segment using a measuring tape. The range and mean of these variables are presented for each species. In addition to these variables, the geographic coordinates and the estimate of incident irradiance (Shading) were taken by canopy photos in each segment, which were analyzed through Image ${ }^{\mathrm{TM}}$ (software that allows quantifying pixel area with presence or absence of canopy cover) and expressed as a percentage to describe each sampling site.
The identification of the macroalgae was carried out with a Zeiss trinocular microscope (model AxioLab.A1 ${ }^{\mathrm{TM}}$ ) equipped with an image capture system (AxioCam). Using a software (ZEN), photomicrographs were obtained to perform ten randomized morphometric measurements of each structure of the populations. For the algae with larger structures, the photomicrographs were obtained with a stereomicroscope (model Zeiss Discovery V.12 ${ }^{\mathrm{TM}}$ ). Macroalgae were identified at the specific level whenever possible, and appropriate references were used for each taxonomic group (e.g. Senna \& Ferreira 1987, Necchi Júnior 1990, Azevedo et al. 1996, Necchi Junior et al. 2001, Skinner \& Entwisle 2001, Branco et al 2002, Kumano 2002, Novis 2004, Bicudo \& Menezes 2006, 

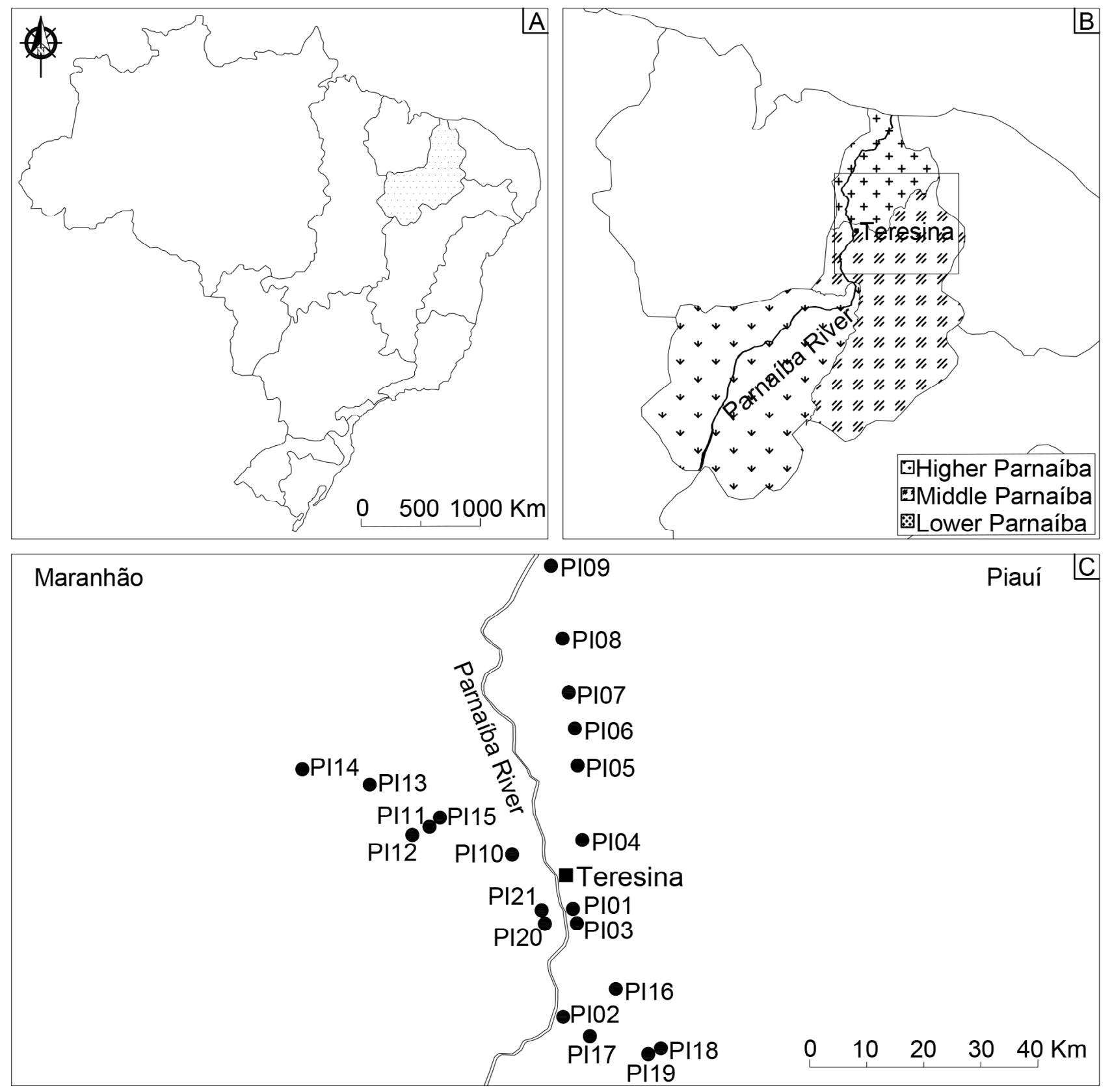

Figure 2. Location map of the Parnaíba basin (A) with its sub-basins and the sampling area (B) showing the distribution of sampling points in relation to Teresina (PI) (C).

Peres \& Branco 2014). The samples were kept in formaldehyde 4\% and deposited in the Herbarium of the Universidade Estadual do Oeste do Paraná (UNOP). New records of the species are marked before the name of each species, single asterisk $(*)$ represent a new record for the Rio Parnaíba basin, two asterisks (**) mean that the species is a new record for the northeastern region of Brazil, and three asterisks $(* * *)$ show that the species is a new record for Brazil.

The following informations are presented: i) descriptions of the species; ii) photomicrographs of the main structures; iii) location of the sampling points; iv) environmental information for each taxon and; v) taxonomic comments (when applicable). The classification system was based in Guiry \& Guiry (2018).

\section{Results}

The taxonomic survey of the macroalgal communities from the Parnaíba River Basin resulted in the identification of 38 taxa in total, of which 32 at a specific level; three vegetative groups of: Spirogyra, Oedogonium and Batrachospermum; two sporophytic stages of red algae: 'Chantransia' macrospora and 'Chantransia' pygmaea and one unidentified species of the genus Phormidium. The best represented taxonomic group was Chlorophyta with 12 taxa (31.6\% of the total), followed by Cyanobacteria with 10 taxa (26.3\%), Bacillariophyta with nine taxa (23.7\%), Rhodophyta with six species (15.8\%) and finally Charophyta with only one taxon sampled (2.6\%). 


\section{PHYLUM BACILLARIOPHYTA \\ CLASS BACILLARIOPHYCEAE ORDER EUNOTIALES}

1.* Eunotia didyma Grunow ex Zimmermann, Broteria, Ser. Bot. 13(2): 51. 1915.

Plate 1. Figure B.

Pseudo-filamentous colonies formed by the union of the frustules by the valvar face; valvar face with biondulate dorsal margin and slightly concave ventral margin, 55.6-75.0 $\mu \mathrm{m}$ long and 10.9-13.4 $\mu \mathrm{m}$ wide; sharp apices and parallel transapical striations; cellular content yellowish-green.

Distribution in the Parnaiba River Basin ( $n=2)$ : Sites PI-10, PI-15. BRAZIL, MARANHÃO: 05³'36.68" S and 42 ${ }^{\circ} 53^{\prime} 38.29$ ' W, $67 \mathrm{amsl}$, 31.V.2017, M.R.Auricchio (UNOP 5790-1); idem, 05²'36.97' S and 43ํ'1'12.97" W, 111 amsl, 31.V.2017, M.R.Auricchio (UNOP 5801-1). Environmental conditions $(n=2)$ : Temp. $26.9-27.6^{\circ} \mathrm{C}\left(\overline{\mathrm{x}}=27.2^{\circ} \mathrm{C}\right)$; Cond. 49-51 $\mu{\mathrm{S} . \mathrm{cm}^{-1}}^{-1}\left(\overline{\mathrm{x}}=50 \mu \mathrm{S} . \mathrm{cm}^{-1}\right) ; \mathrm{pH}$ 6.6-7.3 ( $\left.\overline{\mathrm{x}}=6.9\right)$; Vel. 0.8-1.2 $\mathrm{m} . \mathrm{s}^{-1}\left(\overline{\mathrm{x}}=1 \mathrm{~m} . \mathrm{s}^{-1}\right)$; Depth $11.7-25.2 \mathrm{~cm}(\overline{\mathrm{x}}=18.4 \mathrm{~cm})$; Width $3.0-12.1 \mathrm{~m}$ $(\overline{\mathrm{x}}=7.5 \mathrm{~m})$; Subst. bedrock and boulders; Shading 32-86\% $(\overline{\mathrm{x}}=59 \%)$. Taxonomic comments: the population presented slightly smaller width than described by Costa et al. (2017) and Metzeltin \& Lange-Bertalot (1998), but polymorphism of the species is widely recognized.

2.** Eunotia formica Ehrenberg, Ber. K. Akad. Wiss. Berlin, 414. 1843. Plate 1. Figure C.

Pseudo-filamentous colonies formed by the union of the frustules by the valvar face; valves slightly arcuate with swollen central region, 51.4-105.0 $\mu \mathrm{m}$ in long and 10.1-11.3 $\mu \mathrm{m}$ wide; cuneate or rounded ends; regularly spaced parallel transapical striae; cellular content yellowish-green.

Distribution in the Parnaiba River Basin $(n=3)$ : Sites PI-11, PI-13,

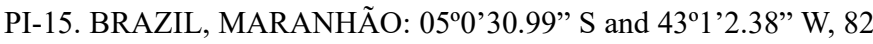
amsl, 31.V.2017, M.R.Auricchio (UNOP 5792-1); idem, 0457'4.608" $\mathrm{S}$ and $43^{\circ} 7^{\prime} 57.21^{\prime}$ 'W, 82 amsl, 31.V.2017, M.R.Auricchio (UNOP 5794); idem, $05^{\circ} 0$ '36.97' S and 43'1'12.97' W, 111 amsl, 31.V.2017, M.R.Auricchio (UNOP 5801).

Environmental conditions $(n=3)$ : Temp. $26.9-27.4^{\circ} \mathrm{C}\left(\overline{\mathrm{x}}=27.1^{\circ} \mathrm{C}\right)$; Cond. 26-49 $\left.\mu{\mathrm{S} . \mathrm{cm}^{-1}}^{(\mathrm{x}}=40.3 \mu \mathrm{S} . \mathrm{cm}^{-1}\right) ; \mathrm{pH}$ 5.8-6.9 $(\overline{\mathrm{x}}=6.4)$; Vel. $0.8-1.2 \mathrm{~m} . \mathrm{s}^{-1}\left(\overline{\mathrm{x}}=0.96 \mathrm{~m} . \mathrm{s}^{-1}\right)$; Depth $20.6-26.6 \mathrm{~cm}(\overline{\mathrm{x}}=24.1 \mathrm{~cm})$; Width 2.4-7.0 m ( $\overline{\mathrm{x}}=4.1 \mathrm{~m})$; Subst. bedrock and sand; Shading 0-87\% $(\overline{\mathrm{x}}=40 \%)$.

3.** Eunotia meridiana Metzeltin \& Lange-Bertalot, Iconogr. Diatomol., 5: 67-68. 1998.

Plate 1. Figure D.

Pseudo-filamentous colonies formed by the union of the frustules by the valve face; arched valves with convex dorsal margin and slightly concave ventral margin, 22.5-34.6 $\mu \mathrm{m}$ long and 6.1-7.7 $\mu \mathrm{m}$ wide; rounded valvar extremity, non-detached from the valvar body; cellular content olive green.

Distribution in the Parnaiba River Basin ( $n=1)$ : Site PI-10. BRAZIL, MARANHÃO: 05'3'36.68' S and 4253'38.29”' W, 67 amsl, 31.V.2017, M.R.Auricchio (UNOP 5790).

Environmental conditions $(n=1)$ : Temp. $27.6^{\circ} \mathrm{C}$; Cond. $51 \mu \mathrm{S} . \mathrm{cm}^{-1}$; pH 7.3; Vel. 0.8 m.s ${ }^{-1}$; Depth 11.7 cm; Width 12.1 m; Subst. bedrock; Shading $86 \%$.
4. **Eunotia transfuga Metzeltin \& Lange-Bertalot, Iconogr. Diatomol. 5: 84-85. 1998.

Plate 1. Figure K.

Pseudo-filamentous colonies of branched chains formed by the union of the ends of the valves; linear, slightly arcuate valves, 175.2-191.1 $\mu \mathrm{m}$ in long and 8.5-11.1 $\mu \mathrm{m}$ in wide, margins with spines; dilated, cuneaterounded and valvar extremities; parallel transapical striations to slightly irradiated towards the extremities; cellular content yellowish-green, filling approximately the entire cell.

Distribution in the Parnaiba River Basin $(n=1)$ : Site PI-13. BRAZIL,

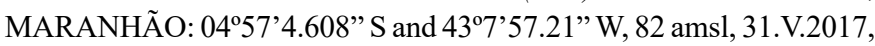
M.R.Auricchio (UNOP 5796-1).

Environmental conditions $(n=1)$ : Temp. $27.5^{\circ} \mathrm{C}$; Cond. $26 \mu \mathrm{S} . \mathrm{cm}^{-1} ; \mathrm{pH}$ 5.8; Vel. 0.8 m.s ${ }^{-1}$; Depth $26.6 \mathrm{~cm}$; Width $7 \mathrm{~m}$; Subst. sand; Shading $87 \%$.

5. **Eunotia xystriformis Manguin, Soc. d'Edi. d'Enseig. Supérieur, p. 49. 1952.

Plate 1. Figure L.

Pseudo-filamentous colonies formed by the adhesion of the frustules through the valve face; linear to slightly curved valves, with slightly swollen median portion, 63.9-89.2 $\mu \mathrm{m}$ in long and 9.0-13.4 $\mu \mathrm{m}$ in diam.; sub-capitated rounded or slightly cuneate extremities; parallel transapical striae; cellular content yellowish-green.

Distribution in the Parnaiba River Basin ( $n=1)$ : Site PI-11. BRAZIL,

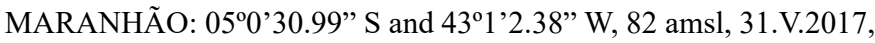
M.R.Auricchio (UNOP 5792).

Environmental conditions ( $n=1)$ : Temp. $27.0^{\circ} \mathrm{C}$; Cond. $46 \mu \mathrm{S} . \mathrm{cm}^{-1}$; pH 6.9; Vel. 0.8 m.s. ${ }^{-1}$; Depth $20.6 \mathrm{~cm}$; Width $2.4 \mathrm{~m}$; Subst. boulders and sand; Shading $0 \%$.

\section{ORDER FRAGILARIALES}

1.** Fragilariforma javanica (Hustedt) C.E.Wetzel, E.Morales \& L.Ector

Plate 1. Figure I-J.

Pseudo-filamentous colonies formed by the diagonal connection of the frustules, forming a zigzag chain, with rare connections by the valve face; linear valves with sub-capitated apices and central region slightly belted, 49.7-62.3 $\mu \mathrm{m}$ long and 5.0-6.9 $\mu \mathrm{m}$ in wide; granulated cell contents yellow-green, filling the whole cell.

Distribution in the Parnaiba River Basin ( $n=1)$ : Site PI-13. BRAZIL,

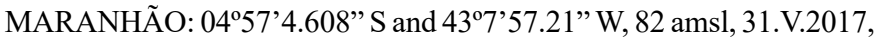
M.R.Auricchio (UNOP 5796).

Environmental conditions $(n=1)$ : Temp. $27.5^{\circ} \mathrm{C}$; Cond. $26 \mu \mathrm{S} . \mathrm{cm}^{-1} ; \mathrm{pH}$ 5.8; Vel. 0.8 m.s ${ }^{-1}$; Depth $26.6 \mathrm{~cm}$; Width $7.0 \mathrm{~m}$; Subst. sand; Shading $87 \%$.

\section{ORDER NAVICULALES}

2.** Diadesmis confervacea Kützing, Die Kiesels. Bacil. oder Diatomeen, p. 109. 1844.

Plate 1. Figure E.

Pseudo-filamentous colonies formed by a connection between frustules by the valvar face; lanceolate valves with sharp to slightly rounded ends, 20.2-24.4 $\mu \mathrm{m}$ long and 6.7-9.7 $\mu \mathrm{m}$ in wide; filiform raphe and rounded central nodule; cellular content yellowish-green. 
Auricchio, M.R. et al.
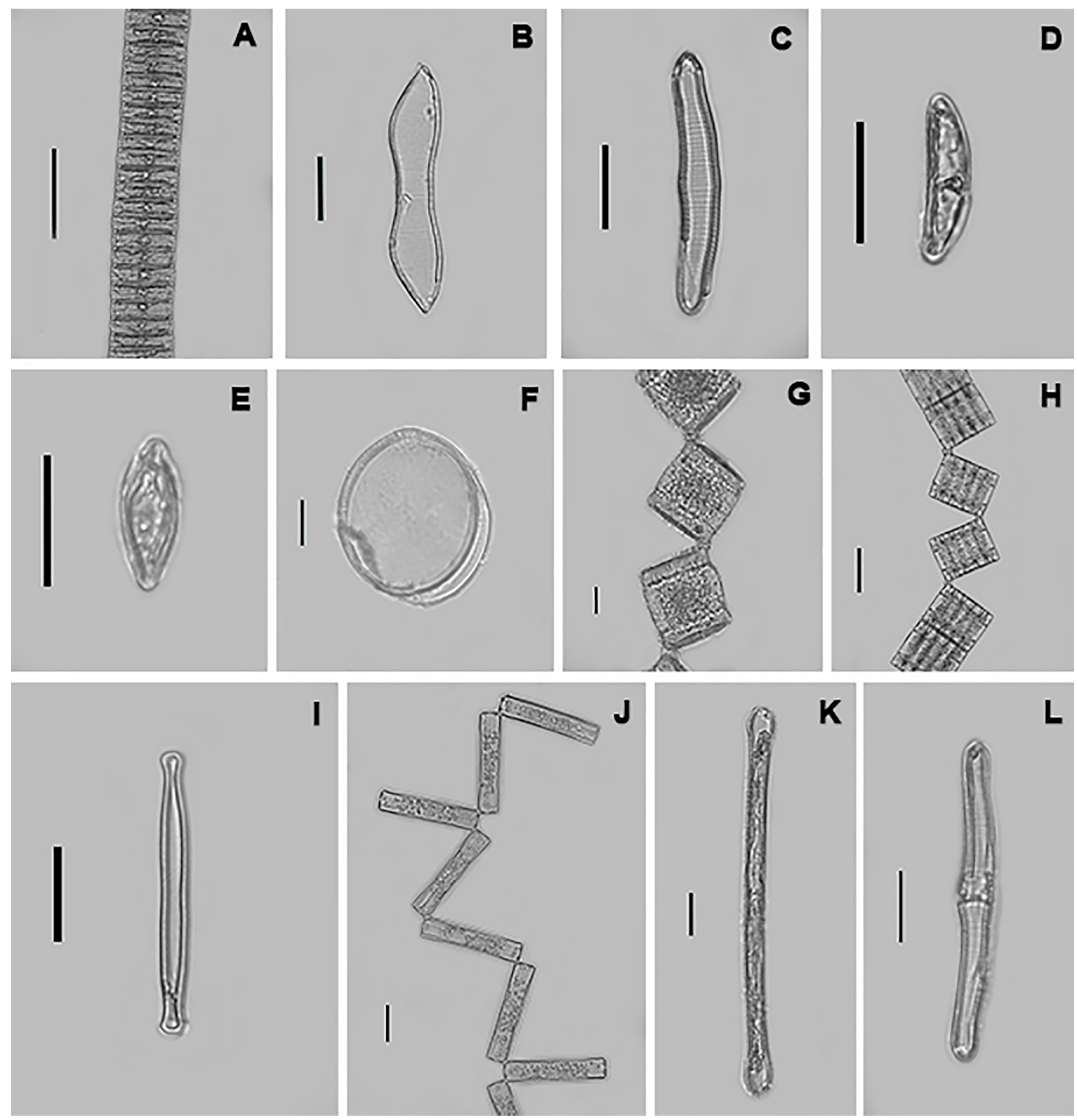

Plate 1. Photomicrographs of the macroalgae species found in the Parnaíba River Basin (PI/MA), Brazil. A. Common view of the Eunotia colonies found as macroalgae in this study. B. Eunotia didyma; C. Eunotia formica; D. Eunotia meridiana; E. Diadesmis cofervacea; F-G. Pleurosira laevis: F. Valvar face showing ocelli; G. General view of the colony; H. Terpsinoe musica; I-J. Fragilariforma javanica: I. Valvar face; J. General view of the colony; K. Eunotia transfuga; L. Eunotia xystriformis. Scale bars $=20 \mu \mathrm{m}$ (Figures B-G, I-L); $100 \mu \mathrm{m}$ (Figure A, H).

Distribution in the Parnaiba River Basin ( $n=1)$ : Site PI-02. BRAZIL, PIAUÍ: $05^{\circ} 19^{\prime} 16.35^{\prime \prime} \mathrm{S}$ and $42^{\circ} 48^{\prime} 29.01^{\prime}$ W, 64 amsl, 29.V.2017, M.R.Auricchio (UNOP 5775-2).

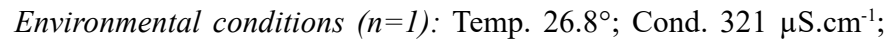
pH 7.8; Vel. 0.5 m.s ${ }^{-1}$; Depth $5 \mathrm{~cm}$; Width $14.4 \mathrm{~m}$; Subst. bedrock; Shading $63 \%$.

\section{CLASS MEDIOPHYCEAE ORDER ANAULALES}

1.* Terpsinoe musica Ehrenberg. Ber. K. Akad. Wiss. Berlin 425. 1843. Plate 1. Figure H.

Pseudo-filamentous colonies forming linear chains by the union of the frustules by the valvar face; valves usually triondulated in valve 
eyesight, (52.7-) 126.5-136.7 $\mu \mathrm{m}$ long and 28.5-46.7 $\mu \mathrm{m}$ wide; silica bars between the ripples in the form of a musical note; cellular content olive green, filling approximately the entire interior of the cell.

Distribution in the Parnaiba River Basin ( $n=1)$ : Site PI-02. BRAZIL, PIAUÍ: 05'19'16.35" S and 42 48'29.01" W, 64 amsl, 29.V.2017, M.R.Auricchio (UNOP 5774-1).

Environmental conditions $(n=1)$ : Temp. 26.8 ; Cond. $321 \mu \mathrm{S} . \mathrm{cm}^{-1}$; pH 7.8; Vel. 0.5 m.s. ${ }^{-1}$; Depth $5 \mathrm{~cm}$; Width $14.4 \mathrm{~m}$; Subst. bedrock; Shading $63 \%$.

Taxonomic comments: the population showed a great variation in length, being possible the existence of another species of Terpsinoe co-occurring in the sample. However, it was not possible to clearly define it using optical microscopy.

\section{ORDER EUPODISCALES}

1.* Pleurosira laevis (Ehrenberg) Compère, Bacillaria 5: 177. 1982.

Plate 1. Figure F-G.

Basionym: Biddulphia laevis Ehrenberg 1843.

Pseudo-filamentous colonies connected by ocelli and forming zigzag chains; cylindrical to subcylindrical frustule; circular to subcircular valves, with a larger diameter of 53.6-76.8 $\mu \mathrm{m}$ and the smaller diameter of 47.6-62.7 $\mu \mathrm{m}$; cellular content olive green to yellow-green.

Distribution in the Parnaiba River Basin ( $n=1)$ : Site PI-02. BRAZIL,

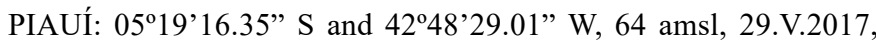
M.R.Auricchio (UNOP 5774-2).

Environmental conditions ( $n=1)$ : Temp. 26.8 ; Cond. $321 \mu \mathrm{S} . \mathrm{cm}^{-1}$; pH 7.8; Vel. 0.5 m.s ${ }^{-1}$; Depth $5 \mathrm{~cm}$; Width $14.4 \mathrm{~m}$; Subst. bedrock; Shading $63 \%$.

\section{PHYLUM CHAROPHYTA \\ CLASS CHAROPHYCEAE \\ ORDER CHARALES}

1.** Nitella furcata (Roxburgh ex Bruzelius) C.Agardh emend., Systema algarum p.124. 1824.

Plate. 2. Figure A-B.

Basionym: Chara furcata W.Roxburgh ex A.Bruzelius 1824.

Plants 9-12 cm high; internodes equal to or longer than branchlets, 1-1.6 cm long; 5-6 monomorphic whorled branchlets, 2-4 furcated, $0.9-1.5 \mathrm{~cm}$ long; 1-3 dactyls, 1-2 celled, abbreviated or elongated, 241.5-801.7 $\mu \mathrm{m}$ long and 84.4-100.9 $\mu \mathrm{m}$ diam., L/D 4.1-8.5; gametangia sessile, present in first and/or second branchlets furcation; 1-2 nucules, 8 convolutions, 328.6-533.8 $\mu \mathrm{m}$ long and 274.2-407.9 $\mu \mathrm{m}$ diam., L/D 0.9-1.3; globules scarce, 208.9-218.7 $\mu \mathrm{m}$ long and 204.9-214.9 $\mu \mathrm{m}$ diam., L/D 1.0-1.1. Distribution in the Parnaiba River Basin $(\mathrm{n}=3)$ : Sites PI-10, PI-14, PI-18. BRAZIL, MARANHÃO: 05'3'36.68' S and 4253'38.29” W, 67 amsl, 31.V.2017, M.R.Auricchio (UNOP 5786); idem, 04 ${ }^{\circ} 55^{\prime} 41.37^{\prime}$ ' S and $43^{\circ} 14^{\prime} 40.48^{\prime \prime}$ W, 98 amsl, 31.V.2017, M.R.Auricchio (UNOP 5798); idem, PIAUÍ: $05^{\circ} 22^{\prime} 27.19^{\prime \prime} \mathrm{S}$ and $42^{\circ} 38^{\prime} 40.05^{\prime}$ W, 71 amsl. 01.VI.2017, M.R.Auricchio (UNOP 5815).

Environmental conditions: $(n=3)$ : Temp. $27.6-29.4^{\circ} \mathrm{C}\left(\overline{\mathrm{x}}=28.2^{\circ} \mathrm{C}\right)$; Cond. $22-79 \mu \mathrm{S}_{\mathrm{cm}}{ }^{-1}\left(\overline{\mathrm{x}}=50.6 \mu \mathrm{S} . \mathrm{cm}^{-1}\right)$; $\mathrm{pH}$ 5.8-7.3 $(\overline{\mathrm{x}}=6.7)$; Vel. $0.11-0.8 \mathrm{~m} . \mathrm{s}^{-1}\left(\overline{\mathrm{x}}=0.3 \mathrm{~m} \cdot \mathrm{s}^{-1}\right)$; Depth $11.3-73.5 \mathrm{~cm}(\overline{\mathrm{x}}=32.1 \mathrm{~cm})$; Width $2.2-12.1 \mathrm{~m}(\overline{\mathrm{x}}=6.4 \mathrm{~m})$; Subst. bedrock and sand; Shading $62-86 \%$ $(\overline{\mathrm{x}}=78 \%)$.

\section{CLASS CONJUGATOPHYCEAE ORDER ZYGNEMATALES}

1.* Spirogyra sp.

Plate 3. Figure C.

Free long unbranched filaments, olivaceous, mostly slippery; cylindrical cells, 78.8-242.5 $\mu \mathrm{m}$ long and 31.7-117.4 $\mu \mathrm{m}$ diam., L/D 0.7-7.0; plastids in spiral parietal bands, with two to five bands; zygospores not observed. Distribution in the Parnaiba River Basin $(n=4)$ : Sites PI-02, PI-10, PI-15, PI-17. BRAZIL, PIAUÍ: 05¹9'16.35" S and 4248'29.01" W, 64 amsl, 29.V.2017, M.R.Auricchio (UNOP 5776); idem, MARANHÃO: 05'3'36.68' S and 42'53'38.29" W, 67 amsl, 31.V.2017, M.R.Auricchio

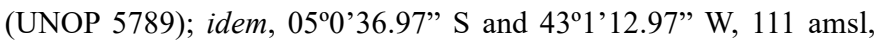
31.V.2017, M.R.Auricchio (UNOP 5801-2); idem, 05'21'20.8' S and 4245'20.91" W, 59 amsl, 01.VI.2017, M.R.Auricchio (UNOP 5809). Environmental conditions $(n=4)$ : Temp. $26.8-29.1^{\circ} \mathrm{C}\left(\overline{\mathrm{x}}=27.6^{\circ} \mathrm{C}\right)$; Cond. 47-321 $\mu \mathrm{S} . c m^{-1}\left(\overline{\mathrm{x}}=183.5 \mu \mathrm{S} . \mathrm{cm}^{-1}\right)$; $\mathrm{pH}$ 6.6-8.0 ( $\left.\overline{\mathrm{x}}=7.4\right)$; Vel. $0.4-1.2 \mathrm{~m} . \mathrm{s}^{-1}\left(\overline{\mathrm{x}}=0.7 \mathrm{~m} . \mathrm{s}^{-1}\right)$; Depth $5.0-25.2 \mathrm{~cm}(\overline{\mathrm{x}}=15.6 \mathrm{~cm})$; Width $2.0-14.4 \mathrm{~m}(\overline{\mathrm{x}}=7.8 \mathrm{~m})$; Subst. bedrock and gravels; Shading $32-83 \%$ $(\overline{\mathrm{x}}=57.2 \%)$.

Taxonomic comments: were found only sterile specimens being impossible to identify them at the specific level.

\section{PHYLUM CHLOROPHYTA}

\section{CLASS CHLOROPHYCEAE} ORDER CHAETOPHORALES

1.** Chaetophora elegans (Roth) C.Agardh, Dispositio Algarum Sueciae, p. 42. 1812.

Plate: 2. Figure C.

Basionym: Rivularia elegans Roth 1802.

Heterotrichous tufts formed by branched filaments immersed in a gelatinous matrix relatively firm, pale green, spherical or hemispherical; basal system composed by prostrate branched filaments with cylindrical cells, producing erect filaments and rhizoids; erect filaments di- or tricotomically branched; lateral branches lax and sparse, fasciculated near the apex; cells of erect system cylindrical, 25.1-63.3 $\mu \mathrm{m}$ long and 4.5-9.1 $\mu \mathrm{m}$ diam., L/D 3.3-9.1; terminal branches densely grouped, tapering at the towards the apex or ending abruptly at an acute apex. Distribution in the Parnaiba River Basin ( $n=1)$ : Site PI-14. BRAZIL, PIAUÍ: $04^{\circ} 55^{\prime} 41.37^{\prime \prime} \mathrm{S}$ and $43^{\circ} 14^{\prime} 40.48^{\prime \prime} \mathrm{W}, 98$ amsl, 31.V.2017, M.R.Auricchio (UNOP 5797).

Environmental conditions: $(n=1)$ : Temp. $27.8^{\circ} \mathrm{C}$; Cond. $22 \mu \mathrm{S} . \mathrm{cm}^{-1}$; pH 5.8; Vel. 0.1 m.s ${ }^{-1}$; Depth $73.5 \mathrm{~cm}$; Width $2.2 \mathrm{~m}$; Subst. gravels; Shading $86 \%$.

2.** Chaetophora pisiformis (Roth) C.Agardh, Dispositio Algarum Sueciae, p. 43. 1812.

Plate 2. Figure D.

Basionym: Rivularia pisiformis Roth 1802.

Heterotrichous tufts formed by branch filaments immersed in a gelatinous matrix relatively firm, pale green, spherical or hemispherical; basal system composed exclusively by rhizoids which arise from basal cells of erect filaments or rarely from median region of filaments; erect filaments di- or tricotomically branched; lateral branches lax and sparse, non fasciculated near the apex; cells of erect system cylindrical, rarely 
Auricchio, M.R. et al.
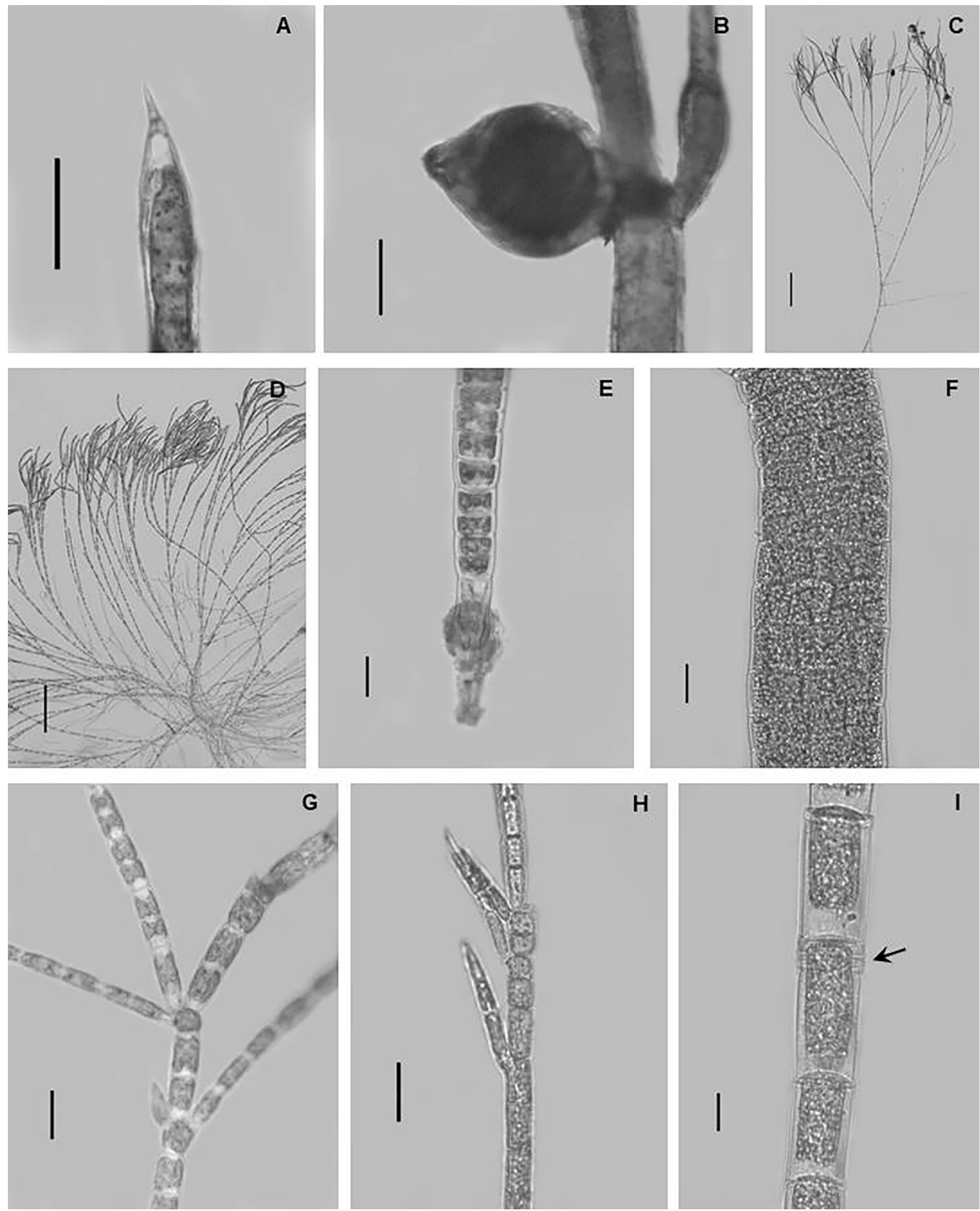

Plate 2. A-B. Nitella furcata: A. Branchlet apex (dactyl); B. Gametangia (oogonia); C. Chaetophora elegans; D. Chaetophora pisiformis; E-F. Schizomeris leiblenii: E. Basal cell; F. Mature multiseriate filament; G. Stigeoclonium amoenum; H. Stigeoclonium helveticum; I. Oedogonium sp. with ring-like caps (arrow). Scale bars $=200 \mu \mathrm{m}$ (Figures A-D); $20 \mu \mathrm{m}$ (Figures E-I). 
Stream macroalgal flora from Parnaíba
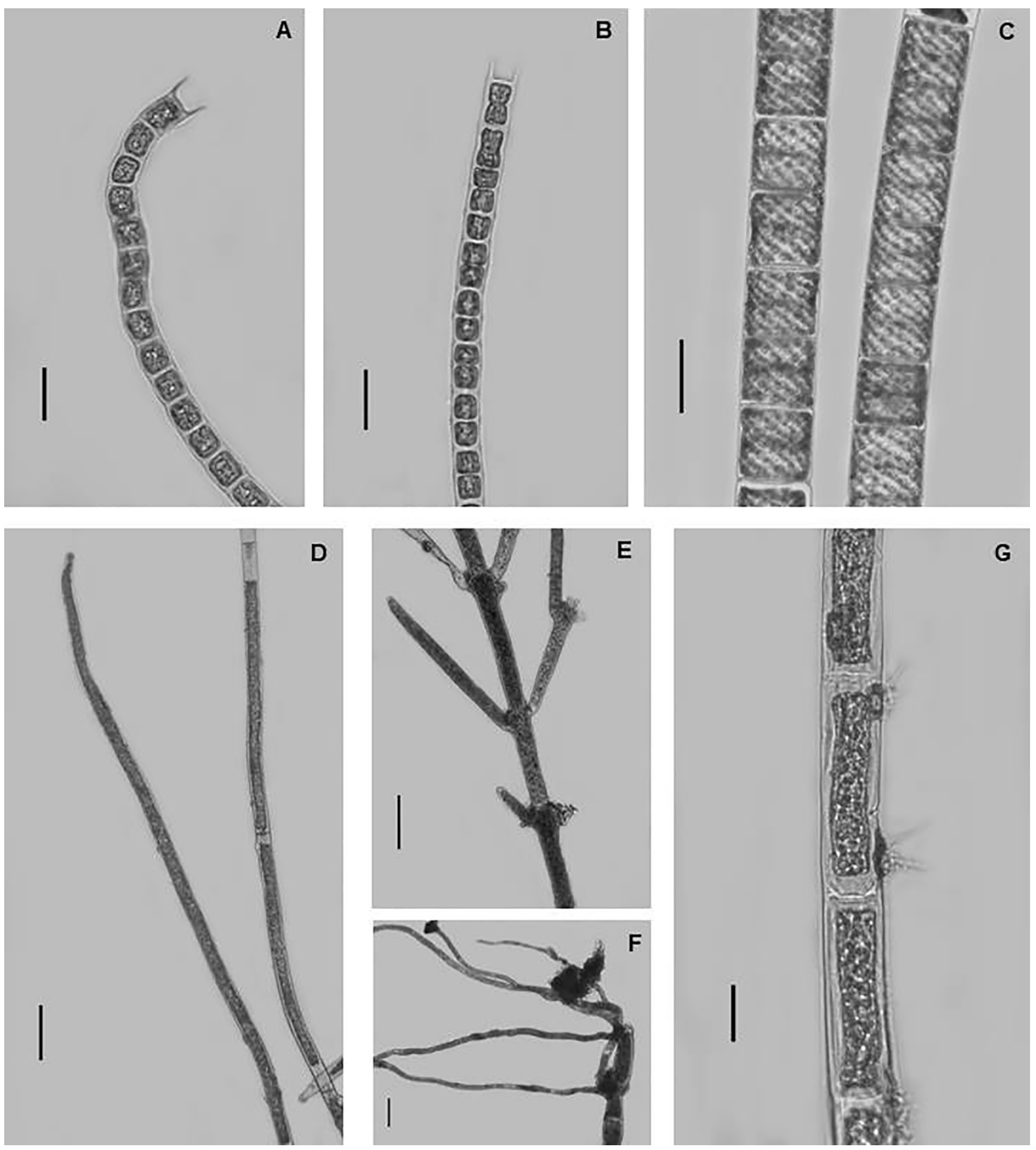

Plate 3. A. Microspora tumidula; B. Microspora willeana; C. Spirogyra sp.; D. Aegagropilopsis sterrocladia; E-F. Cladophora glomerata: E. Branched filamento; F. Rhizoids; G. Rhizoclonium hieroglyphicum. Scale bars $=20 \mu \mathrm{m}$ (Figures A-B, G); $100 \mu \mathrm{m}$ (Figure C, E-F); 200 $\mu \mathrm{m}$ (Figure D).

inflated 12.9-58.3 $\mu \mathrm{m}$ long and 5.2-11.2 $\mu \mathrm{m}$ diam., L/D 1.9-6.4; branches ending abruptly at an acute or rounded apex.

Distribution in the Parnaiba River Basin ( $n=3)$ : Sites PI-15, PI-16,

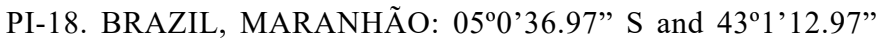
W, 111 amsl, 31.V.2017, M.R.Auricchio (UNOP 5804); idem, PIAUÍ: $05^{\circ} 16^{\prime} 33.73$ ' S and 42 42 '53.23” W, 38 amsl, 01.VI.2017,

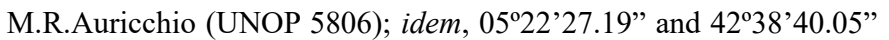
W, 71 amsl, 01.VI.2017, M.R.Auricchio (UNOP 5816).

Environmental conditions: $(n=3)$ : Temp. $26 \cdot 9-29 \cdot 4^{\circ} \mathrm{C}\left(\overline{\mathrm{x}}=28^{\circ} \mathrm{C}\right)$; Cond.

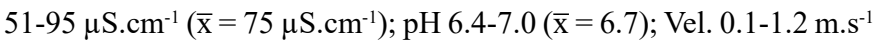
$\left(\overline{\mathrm{x}}=0.5 \mathrm{~m} . \mathrm{s}^{-1}\right)$; Depth $11.3-29.0 \mathrm{~cm}(\overline{\mathrm{x}}=21.8 \mathrm{~cm})$; Width $2.7-5.1 \mathrm{~m}(\overline{\mathrm{x}}$ $=3.6 \mathrm{~m})$; Subst. boulders and sand; Shading $32-62 \%(\overline{\mathrm{x}}=47 \%)$. 
3.** Schizomeris leibleinii Kützing, Phycologia Generalis, p. 247. 1843. Plate: 2. Figures E-F.

Isolated unbranched filaments with slight constrictions in regular interval, olivaceous; basal region attached to substratum by a basal rhizoid-like cell, 28.4-48.3 $\mu \mathrm{m}$ long and 13.4-25.0 $\mu \mathrm{m}$ diam., L/D 1.32.8; basal portion uniseriate, cylindrical cells, 5.8-25.9 $\mu \mathrm{m}$ long and 9.9-14.8 $\mu \mathrm{m}$ diam., L/D 0.3-1.0; middle and apical portion multiseriate with quadratic cell in evident rows, 8.3-18.9 $\mu \mathrm{m}$ long and 10.3-31.4 $\mu \mathrm{m}$ diam., L/D 0.4-1.0; apical cell rounded to acute; parietal plastids, forming a band in uniseriate region and perforated in multiseriate region. Distribution in the Parnaiba River Basin $(n=1)$ : Site PI-03. BRAZIL, PIAUÍ: 05 9'2.484"S and 4248'6.444” W, 59 amsl, 29.V.2017, M.R.Auricchio (UNOP 5777-1).

Environmental conditions $(n=1)$ : Temp. $32.3^{\circ} \mathrm{C}$; Cond. $432 \mu \mathrm{S} . \mathrm{cm}^{-1}$; pH 7.3; Vel. 0.4 m.s ${ }^{-1}$; Depth $6.6 \mathrm{~cm}$; Width $2.1 \mathrm{~m}$; Subst. gravels; Shading $0 \%$.

4.** Stigeoclonium amoenum Kützing, Phycologia Germanica, p. 198. 1845.

Plate: 2. Figure G.

Heterotrichous tufts formed by branched filaments, bright green; well developed erect system and reduced prostrate system, composed exclusively of profuse mass of rhizoids emerging from the basal part of erect filaments; main axis differentiated in short and long cells; short cells cylindrical, quadratic to slightly inflated, 4.8-14.0 $\mu \mathrm{m}$ long and 6.5-13.5 $\mu \mathrm{m}$ diam., L/D 0.6-1.2; long cells cylindrical, 6.9-23.8 $\mu \mathrm{m}$ long and 5.4-13.8 $\mu \mathrm{m}$ diam, L/D 0.7-1.5; single, parietal chloroplast; branches alternate or opposite, often with two or three branches from the same short cell, lateral branches formed by cylindrical cells; apex of main branches acuminate with occasional multicellular hyaline hairs. Distribution in the Parnaiba River Basin $(n=1)$ : Site PI-03. BRAZIL, PIAUÍ: 05'9'2.484" S and 4248'6.444” W, 59 amsl, 29.V.2017, M.R.Auricchio (UNOP 5777).

Environmental conditions $(n=1)$ : Temp. $32.3^{\circ} \mathrm{C}$; Cond. $432 \mu \mathrm{S} . \mathrm{cm}^{-1}$; pH 7.3; Vel. 0.4 m.s ; $^{-1}$ Depth $6.6 \mathrm{~cm}$; Width $2.1 \mathrm{~m}$; Subst. gravels; Shading $0 \%$.

Taxonomic comments: the morphometric measurements are slightly lower than those described in Branco et al. (2002) to Brazilian streams, however, all morphological characteristics agree with that described for this species.

5.** Stigeoclonium helveticum Vischer, Beihefte zum Botanischen Centralblatt 51: 56. 1933.

Plate 2. Figure H.

Heterotrichous tufts formed by branched filaments, bright green; well developed erect system and reduced prostrate system, composed exclusively of profuse mass of short rhizoids emerging from the erect filaments; axial cells cylindrical, similar in shape and size, 5.0-10.6 $\mu \mathrm{m}$ long and 5.8-10.6 $\mu \mathrm{m}$ diam., L/D 0.5-1.3; single parietal chloroplast; alternate branches and rarely opposite or with multiples branches; branch cells, 12.6-28.9 $\mu \mathrm{m}$ long and 4.2-8.0 $\mu \mathrm{m}$ diam., L/D 1.9-6.0; apex of main branches acuminate with occasional multicellular hyaline hairs. Distribution in the Parnaiba River Basin $(n=1)$ : Site PI-21. BRAZIL, MARANHÃO: $05^{\circ} 9^{\prime} 32.76^{\prime} \mathrm{S}$ and 42 50'16.44" W, $69 \mathrm{amsl}$, 02.VI.2017, M.R.Auricchio (UNOP 5822).
Environmental conditions $(n=1)$ : Temp. $30.0^{\circ} \mathrm{C}$; Cond. $48 \mu{\mathrm{S} . \mathrm{cm}^{-1}}^{-1} \mathrm{pH}$ 7.3; Vel. 0.05 m.s s $^{-1}$ Depth $21 \mathrm{~cm}$; Width $5.4 \mathrm{~m}$; Subst. sand; Shading $0 \%$.

\section{ORDER OEDOGONIALES \\ 1. ** Oedogonium $\mathrm{sp}$.}

Plate 2. Figure I.

Entangled unbranched filaments, olivaceous to brown; cylindrical cells, slightly constricted in septa, 25.9-174.9 $\mu \mathrm{m}$ long and 10.4-49.3 $\mu \mathrm{m}$ diam., L/D 1.1-4.0; chloroplast parietal, netlike with several pyrenoids; sometimes with ring-like caps near the cross wall; oogonia and antheridia not observed.

Distribution in the Parnaiba River Basin ( $n=2)$ : Sites PI-10, PI-13. BRAZIL, MARANHÃO: 05³'36.68' S and 42'53'38.29" W, 67 amsl, 31.V.2017, M.R.Auricchio (UNOP 5790-2); idem, 04 ${ }^{\circ} 57^{\prime} 4.608^{\prime \prime} \mathrm{S}$ and 4307'57.21" W, 82 amsl, 31.V.2017, M.R.Auricchio (UNOP 5794-1). Environmental conditions $(n=2)$ : Temp. $27.4-27.6^{\circ} \mathrm{C}\left(\overline{\mathrm{x}}=27.5^{\circ} \mathrm{C}\right)$; Cond. 26-51 $\mu \mathrm{S} . c m^{-1}\left(\overline{\mathrm{x}}=38.5 \mu \mathrm{S} . \mathrm{cm}^{-1}\right) ; \mathrm{pH}$ 5.8-7.3 ( $\left.\overline{\mathrm{x}}=6.5\right)$; Vel. 0.8 m. $\mathrm{s}^{-1}$; Depth 11.7-26.6 cm $(\overline{\mathrm{x}}=19.1 \mathrm{~cm})$; Width 7.0-12.1 m $(\overline{\mathrm{x}}=9.5 \mathrm{~m})$; Subst. bedrock and sand; Shading $86-87 \%(\overline{\mathrm{x}}=86.5 \%)$.

Taxonomic comments: were found only sterile specimens, being impossible to identify them at the specific level.

\section{ORDER SPHAEROPLEALES}

1.** Microspora tumidula Hazen, Memoirs of the Torrey Botanical Club 11: 177. 1902.

Plate 3. Figure A.

Entangled unbranched filaments, constricted in septa with evident $\mathrm{H}$ pieces; cylindrical cells, 9.7-15.2 $\mu \mathrm{m}$ long and 8.8-11.2 $\mu \mathrm{m}$ diam., L/D 0.9-1.4; cell wall thin to moderately thick, $0.5-1.0 \mu \mathrm{m}$ diam.; plastids reticulated, completely surrounding the cell lumen.

Distribution in the Parnaiba River Basin $(n=2)$ : Sites PI-12, PI-13. BRAZIL, PIAUÍ: S 05¹'52.82" S and 433'35.02" W; 126 amsl. 31.V.2017, M.R.Auricchio (UNOP 5793); idem, MARANHÃO: 04 $57^{\circ} 4.608^{\prime \prime} \mathrm{S}$ and $43^{\circ} 7^{\prime} 57.21^{\prime \prime} \mathrm{W}, 82$ amsl. 31.V.2017, M.R.Auricchio (UNOP 5795-1).

Environmental conditions $(n=2)$ : Temp. $27.4-27.6^{\circ} \mathrm{C}\left(\overline{\mathrm{x}}=27.5^{\circ} \mathrm{C}\right)$; Cond. $24-26 \mu$ S.cm ${ }^{-1}\left(\bar{x}=25 \mu\right.$ S.cm $\left.{ }^{-1}\right) ; \mathrm{pH}$ 5.4-5.8 ( $\left.\overline{\mathrm{x}}=5.6\right)$; Vel. 0.3-0.8 $\mathrm{m} . \mathrm{s}^{-1}\left(\overline{\mathrm{x}}=0.5 \mathrm{~m} . \mathrm{s}^{-1}\right)$; Depth $18.0-26.6 \mathrm{~cm}(\overline{\mathrm{x}}=22.3 \mathrm{~cm})$; Width $2.3-7.0 \mathrm{~m}$ $(\overline{\mathrm{x}}=4.6 \mathrm{~m})$; Subst. sand and macrophytes; Shading $84-87 \%(\overline{\mathrm{x}}=85 \%)$.

2.** Microspora willeana Lagerheim, Berichte der Deutschen Botanischen Gesellschaft 5: 414. 1887.

Plate 3. Figure B.

Entangled unbranched filaments, non or constricted in septa with non-evident $H$ pieces; cylindrical cells, 9.1-178 $\mu \mathrm{m}$ long and 9.3-11.9 $\mu \mathrm{m}$ diam., L/D 0.8-1.8; cell wall thin to moderately thick, 0.5-1.2 $\mu \mathrm{m}$ diam.; plastids in perforated plate, completely surrounding cell lumen. Distribution in the Parnaiba River Basin ( $n=4)$ : Sites P-06, PI-07, PI-12, PI-13. BRAZIL, PIAUÍ: 04'50'57.66" S and 4247'46.89” W, 90 amsl, 30.V.2017, M.R.Auricchio (UNOP 5783); idem, 04²4'21.22" S and 42 $48^{\prime} 27.18^{\prime \prime} \mathrm{W}, 79$ amsl, 30.V.2017, M.R.Auricchio (UNOP 5784); idem, MARANHÃO: $05^{\circ} 1^{\prime} 52.82^{\prime \prime} \mathrm{S}$ and 43³'35.02" W, 126 amsl, 31.V.2017, M.R.Auricchio (UNOP 5793); idem, 0457'4.608” S and $43^{\circ} 7^{\prime}$ 57.21' 'W, 82 amsl, 31.V.2017, M.R.Auricchio (UNOP 5795). Environmental conditions $(n=4)$ : Temp. $27.4-28.7^{\circ} \mathrm{C}\left(\overline{\mathrm{x}}=28.0^{\circ} \mathrm{C}\right)$; Cond. 24-492 $\mu{\mathrm{S} . \mathrm{cm}^{-1}}^{-1}\left(\overline{\mathrm{x}}=192 \mu \mathrm{S} . \mathrm{cm}^{-1}\right) ; \mathrm{pH}$ 5.4-6.1 $(\overline{\mathrm{x}}=5.8)$; Vel. 
0.1-0.8 m.s $\mathrm{s}^{-1}\left(\overline{\mathrm{x}}=0.4 \mathrm{~m} . \mathrm{s}^{-1}\right)$; Depth 5.2-26.6 cm $(\overline{\mathrm{x}}=14.0 \mathrm{~cm})$; Width 0.4-7.0 $\mathrm{m}(\overline{\mathrm{x}}=3.5 \mathrm{~m})$; Subst. sand and macrophytes; Shading $18-88 \%$ $(\overline{\mathrm{x}}=69 \%)$.

\section{CLASS ULVOPHYCEAE}

\section{ORDER CLADOPHORALES}

1.** Aegagropilopsis sterrocladia (Skuja) Boedeker, Journal of Phycology 48(3): 822. 2012.

Plate 3. Figure D.

Basionym: Cladophora sterrocladia Skuja 1949.

Tufts of long branched filaments, olivaceous; multinucleate cells; fixed in substrate by primary or secondary rhizoids; principal axis poorly branched, only primary branches, elongated, generally unilateral; lateral branches insert in apical or slightly subterminal cellular pole; axis cells cylindrical, 363.3-1194.9 $\mu \mathrm{m}$ long and 49.7-63.1 $\mu \mathrm{m}$ diam., L/D 6.0-18.9; apical cells very longer than axis cells, 1261.2-3289.9 long and 52.3-58.7 diam., L/D 22.7-60.2; zoosporangia not observed. Distribution in the Parnaiba River Basin $(n=1)$ : Site PI-10. BRAZIL, MARANHÃO: 05'3'36.68' S and 4253'38.29”' W, 67 amsl, 31.V.2017, M.R.Auricchio (UNOP 5788).

Environmental conditions $(n=1)$ : Temp. $27.6^{\circ} \mathrm{C}$; Cond. $51 \mu \mathrm{S} . \mathrm{cm}^{-1}$; pH 7.3; Vel. 0.8 m.s ${ }^{-1}$; Depth 11.7 cm; Width $12.1 \mathrm{~m}$; Subst. bedrock; Shading $86 \%$.

Taxonomic comments: the diameter of the cells was slightly narrower and the apical cell slightly larger than that described by Peres \& Branco (2014, as Cladophora sterrocladia) (axis cell diameter: 61.0-137.7 $\mu \mathrm{m}$; apical cell length: 778.0-2045.3 $\mu \mathrm{m}[-2413.0 \mu \mathrm{m}]$, apical cell width: 62.6-109.9 $\mu \mathrm{m}$ ) for the Brazilian material.

2. Cladophora glomerata (Linnaeus) Kützing, Phycologia generalis oder Anatomie, Physiologie und Systemkunde der Tange: 266. 1843. Plate 3. Figure E-F.

Basionym: Conferva glomerata Linnaeus 1753.

Tufts of branched filaments, brownish; multinucleate cells; profusely branched, branches inserted laterally or obliquely in apical portion of cell, alternated, unilateral or opposite; axial cells cylindrical, 164.0$412.8 \mu \mathrm{m}$ long and 27.7-41.6 $\mu \mathrm{m}$ diam., L/D 5.5-12.8; apical cells longer than axis cells, 87.8-1660.3 $\mu \mathrm{m}$ long and 26.5-38.9 $\mu \mathrm{m}$ diam., L/D 14,3-42,6.

Distribution in the Parnaiba River Basin ( $n=2)$ : Sites PI-02, PI-17. BRAZIL, PIAUÍ: $05^{\circ} 19^{\prime} 16.35^{\prime \prime} \mathrm{S}$ and $42^{\circ} 48^{\prime} 29.01^{\prime \prime} \mathrm{W}, 64 \mathrm{amsl}$, 29.V.2017, M.R.Auricchio (UNOP 5772); idem, 05²1'20.8' S and 42 45'20.91" W, 59 amsl, 01.VI.2017, M.R.Auricchio (UNOP 5813). Environmental conditions $(n=2)$ : Temp. $26.8-29.1^{\circ} \mathrm{C}\left(\overline{\mathrm{x}}=27.9^{\circ} \mathrm{C}\right)$; Cond. 312-321 $\mu{\mathrm{S} . \mathrm{cm}^{-1}}\left(\overline{\mathrm{x}}=316 \mu \mathrm{S} . \mathrm{cm}^{-1}\right)$; $\mathrm{pH}$ 7.8-8.0 ( $\left.\overline{\mathrm{x}}=7.9\right)$; Vel. $0.4-0.5 \mathrm{~m} . \mathrm{s}^{-1}\left(\overline{\mathrm{x}}=0.5 \mathrm{~m} . \mathrm{s}^{-1}\right)$; Depth $5.0-20.6 \mathrm{~cm}(\overline{\mathrm{x}}=12.8 \mathrm{~cm})$; Width $2.0-14.4 \mathrm{~m}(\overline{\mathrm{x}}=8.2 \mathrm{~m})$; Subst. bedrock and boulders; Shading $48-63 \%$ $(\overline{\mathrm{x}}=55 \%)$.

3.** Rhizoclonium hieroglyphicum (C.Agardh) Kützing, Phycologia germanica: 206. 1845.

Plate 3. Figure G.

Basionym: Conferva hieroglyphica C.Agardh 1827.

Long unbranched filaments, flexuous; multinucleate cells; cylindrical cells, 150.0-289.0 $\mu \mathrm{m}$ long and 85.3-95.7 $\mu \mathrm{m}$ diam., L/D 1.6-3.1, cell wall narrow to thick and stratified, 2.8-4.0 $\mu \mathrm{m}$ diam.; plastids net-like with several pyrenoids; sporangia not observed.

Distribution in the Parnaiba River Basin ( $n=1)$ : Site PI-17. BRAZIL, PIAUÍ: 05'21'20.8” S and 42 45'20.91” W, 59 amsl, 01.VI.2017, M.R.Auricchio (UNOP 5810).

Environmental conditions $(n=1)$ : Temp. $29.1^{\circ} \mathrm{C}$; Cond. $312 \mu$ S.cm ${ }^{-1} ; \mathrm{pH}$ 8.0; Vel. 0.4 m.s ${ }^{-1}$; Depth $20.6 \mathrm{~cm}$; Width $2 \mathrm{~m}$; Subst. boulders, pebbles and sand; Shading $48 \%$.

\section{PHYLUM CYANOPHYTA}

\section{CLASS CYANOPHYCEAE}

\section{ORDER OSCILLATORIALES}

1.* Geitlerinema splendidum (Greville ex Gomont) Anagnostidis, Plant Syst. And Evolut. 164: 43. 1989.

Plate 4. Figure A.

Basionym: Oscillatoria splendida Greville ex Gomont 1892.

Mats of filaments, bluish green; trichomes straight or bent, distinctly attenuated at the apex (with proboscis), unconstricted; granulated septa, one or two granules each side; intercalar cells longer than wide, 3.1-4.4 $\mu \mathrm{m}$ long and 1.3-2.6 $\mu \mathrm{m}$ diam., L/D 1.4-2.4; apical cell capitate, (6.9) 9.7-14.5 $\mu \mathrm{m}$ long and 1.0-2.2 $\mu \mathrm{m}$ diam., L/D (3.6) 6.5-11.3; cellular content homogeneous, blue-green.

Distribution in the Parnaiba River Basin $(n=4)$ : Sites PI-11, PI-15, PI16, PI-19. BRAZIL, MARANHÃO: 050'30.99" S and 431'2.38” W, 82 amsl, 31.V.2017, M.R.Auricchio (UNOP 5791); idem, 050'36.97" S and 43'1'12.97' W, 111 amsl, 31.V.2017, M.R.Auricchio (UNOP 5803); idem, PIAUÍ: $05^{\circ} 16^{\prime} 33.73$ " S and 42² '53.23" W, 38 amsl, 01.VI.2017, M.R.Auricchio (UNOP 5808-2); idem, 05'22'45.37' S and 42³9'1.548 W, 68 amsl, 01.VI.2017, M.R.Auricchio (UNOP 5820).

Environmental conditions $(n=4)$ : Temp. $26.9-29.4^{\circ} \mathrm{C}\left(\overline{\mathrm{x}}=27.7^{\circ} \mathrm{C}\right)$; Cond. $46-130 \mu \mathrm{S} . \mathrm{cm}^{-1}\left(\overline{\mathrm{x}}=80 \mu \mathrm{S} . \mathrm{cm}^{-1}\right) ; \mathrm{pH}$ 6.4-6.9 ( $\left.\overline{\mathrm{x}}=6.6\right)$; Vel. 0.2-1.2 $\mathrm{m} . \mathrm{s}^{-1}\left(\overline{\mathrm{x}}=0.7 \mathrm{~m} . \mathrm{s}^{-1}\right)$; Depth $17.6-29.0 \mathrm{~cm}(\overline{\mathrm{x}}=23.1 \mathrm{~cm})$; Width $2.1-3.0$ $\mathrm{m}(\overline{\mathrm{x}}=2.5 \mathrm{~m})$; Subst. boulders and sand; Shading $0-48 \%(\overline{\mathrm{x}}=32 \%)$.

2.* Lyngbya aff. majuscula Harvey ex Gomont, Annales des Sciences Naturelles, Botanique, Série 7 16: 131. 1892.

Plate 4. Figure B.

Mats of filaments, dark yellowish green; thick and hyaline sheath, 3.3$5.7 \mu \mathrm{m}$; trichomes cylindrical, 46.4-51.4 $\mu \mathrm{m}$ diam., unconstricted in septa; intercalar cells short, discoids, shorter than long, 3.5-8.3 $\mu \mathrm{m}$ long and 36.6-46.7 $\mu \mathrm{m}$ diam., L/D 0.08-0.1; apical cells rounded, without calyptra, 6.9-10.4 $\mu \mathrm{m}$ long and 30.4-39.0 $\mu \mathrm{m}$ diam., L/D 0.1-0.2; cellular content homogeneous, olivaceous to dark reddish-green.

Distribution in the Parnaiba River Basin $(n=1)$ : Site PI-17. BRAZIL, PIAUÍ: $05^{\circ} 21^{\prime} 20.8^{\prime} \mathrm{S}$ and 42 45'20.91” W, 59 amsl, 01.VI.2017, M.R.Auricchio (UNOP 5811).

Environmental conditions $(n=1)$ : Temp. $29.1^{\circ} \mathrm{C}$; Cond. $316 \mu \mathrm{S} . \mathrm{cm}^{-1}$; pH 8.0; Vel. 0.4 m.s ${ }^{-1}$; Depth $20.6 \mathrm{~cm}$; Width $2.0 \mathrm{~m}$; Subst. boulders; Shading $48 \%$.

Taxonomic comments: According to Komárek \& Anagnostidis (2005), L. majuscula is a typical species of marine environments. However, the identification of this material is consistent with the description proposed by the same authors. In addition, other authors have already described the species in freshwater environments (e.g. Desikachary 1959) and, for this reason, we maintained the identification. In spite of this, we consider that the environmental variation between freshwater 
Auricchio, M.R. et al.
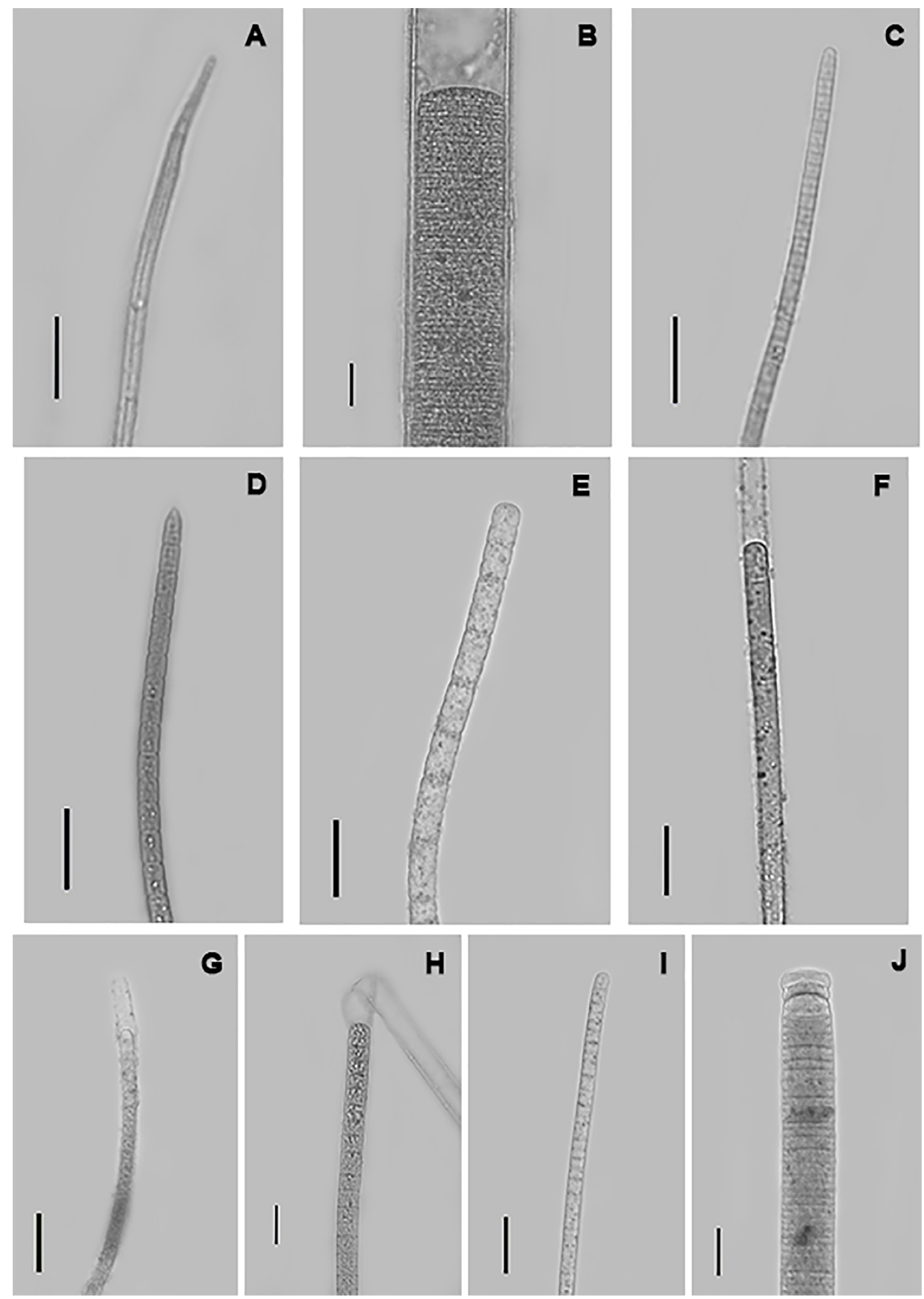

Plate 4. A. Geitlerinema splendidum; B. Lyngbya aff. majuscula; C. Microcoleus autumnalis; D. Microcoleus lacustris; E. Phormidium aerugineocaeruleum; F. Phormidium retzii; G. Phormidium corium; H. Phormidium sp.; I. Phormidium tergestinum; J. Oscillatoria princeps. Scale bars $=10 \mu \mathrm{m}$ (Figure A); $20 \mu \mathrm{m}$ (Figures B-J). 
and marine environments probably selects lineages with very different physiological characteristics. Thus, future studies involving molecular biology may clearly support a new taxonomic configuration.

3.** Microcoleus autumnalis (Gomont) Strunecky et al., Journal of Phycol. 49(6): 1176. 2013.

Plate 4. Figure C.

Basionym: Phormidium autumnale Gomont 1892.

Mats of filaments, greenish blue; trichomes with cellular content homogeneous, unconstricted and granulated septa; intercalar cells wider than long, 1.9-3.8 $\mu \mathrm{m}$ long and 3.4-4.4 $\mu \mathrm{m}$ diam., L/D 0.5-0.9; apical cell rounded to capitate, 3.3-5.9 $\mu \mathrm{m}$ long and 2.4-3.9 $\mu \mathrm{m}$ diam., L/D 0.9-2.0, cellular content homogeneous, blue-green.

Distribution in the Parnaiba River Basin ( $n=1)$ : Site PI-21. BRAZIL, MARANHÃO: 05'9'32.76" S and 42 50'16.44" W, 69 amsl, 02.VI.2017, M.R.Auricchio (UNOP 5821).

Environmental conditions $(n=1)$ : Temp. $30.0^{\circ} \mathrm{C}$; Cond. $48 \mu \mathrm{S} . \mathrm{cm}^{-1}$; pH 7.3; Vel. 0.05 m.s ${ }^{-1}$; Depth $21.0 \mathrm{~cm}$; Width $5.4 \mathrm{~m}$; Subst. sand; Shading $0 \%$.

4.*** Microcoleus lacustris Farlow ex Gomont, Annales des Sciences Naturelles, Botanique, Série 7, 15: 359. 1892.

Plate 4. Figure D.

Mats of filaments, dark greenish blue; filaments formed by one to various trichomes in a wider hyaline sheath; sheath open in apex, with free trichomes; trichomes cylindrical, constricted in septa; intercalar cells granulated, quadratic to longer than wide, 4.1-9.4 $\mu \mathrm{m}$ long and 4.5-6.2 $\mu \mathrm{m}$ diam., L/D 0.8-1.8; apical cells no capitate, conical rounded, 6.2-9.5 $\mu \mathrm{m}$ long and 3.7-5.5 $\mu \mathrm{m}$ diam., L/D 1.4-2.3; cellular content granulated, dark greenish blue.

Distribution in the Parnaiba River Basin ( $n=1)$ : Site PI-16. BRAZIL, PIAUÍ: 05 ${ }^{\circ} 16^{\prime} 33.73$ " and 42 42 '53.23" W, 95 amsl, 01.VI.2017, M.R.Auricchio (UNOP 5808).

Environmental conditions $(n=1)$ : Temp. $27.8^{\circ} \mathrm{C}$; Cond. $95 \mu \mathrm{S} . \mathrm{cm}^{-1}$; pH 6.4; Vel. 0.1 m.s. ${ }^{-1}$; Depth $29 \mathrm{~cm}$; Width 2,7 m; Subst. gravels and sand; Shading $48 \%$.

Taxonomic comments: the identification is consistent with the one proposed by Komárek \& Anagnostidis (2005) for M. lacustris, however, in his description, the species has no granulation as reported here.

5.* Oscillatoria princeps Vaucher ex Gomont, Annales des Sciences Naturelles, Botanique, Série 7 16: 206. 1892.

Plate 4. Figure J.

Mats of filaments, dark green; sheath absent; trichomes cylindrical, unconstricted; intercalar cells discoids, very wider than long, 2.9-7.5 $\mu \mathrm{m}$ long and 22.2-46.1 $\mu \mathrm{m}$ diam., L/D 0.08-0.2; apical cell truncate, sometimes with a distinct thickening in cell wall, 5.0-10.5 $\mu \mathrm{m}$ long and 20.9-33.4 $\mu \mathrm{m}$ diam., L/D 0.1-0.3; cellular content homogeneous, olivaceous to dark green.

Distribution in the Parnaiba River Basin $(n=1)$ : Site PI-04. BRASIL, PIAUÍ: 05 ${ }^{\circ} 1$ '58.87' S and 42 46'41.44" W, 43 amsl, 30.V.2017, M.R.Auricchio (UNOP 5780).

Environmental conditions $(n=1)$ : Temp. $28.1^{\circ} \mathrm{C}$; Cond. $301 \mu \mathrm{S} . \mathrm{cm}^{-1}$; pH 6.6; Vel. 0.9 m.s ${ }^{-1}$; Depth 5.4 cm; Width 19.0 m; Subst. bedrock; Shading $31 \%$.
6.** Phormidium aerugineocaeruleum (Gomont) Anagnostidis \& Komárek, Algolog. Stud., 50-53: 407. 1988.

Plate 4. Figure E.

Basionym: Lyngbya aerugineo-caerulea Gomont 1892.

Mats of filaments, greenish blue to dark blue; sheath thin, hyaline, homogeneous, generally absent; trichomes unconstricted in septa or rarely with weak constriction; intercalar cells wider than long, 4.2-6.1 $\mu \mathrm{m}$ long and 5.6-7.4 $\mu \mathrm{m}$ diam., L/D 0.6-0.8; apical cells rounded, 3.3-5.4 $\mu \mathrm{m}$ long and 5.0-6.5 $\mu \mathrm{m}$ diam., L/D 0.5-1.1; cellular content granulated, bright blue-green, granules greenish, small and large.

Distribution in the Parnaiba River Basin $(n=1)$ : Site PI-09. BRAZIL, PIAUÍ: 04'34'46.12" S and 42 ${ }^{\circ} 50^{\prime} 24.72 ” \mathrm{~W}, 55$ amsl, 30.V.2017, M.R.Auricchio (UNOP 5785).

Environmental conditions $(n=1)$ : Temp. $30.2^{\circ} \mathrm{C}$; Cond. $54 \mu \mathrm{S} . \mathrm{cm}^{-1}$; pH 6.7; Vel. 3.3 m.s. ${ }^{-1}$; Depth $15.0 \mathrm{~cm}$; Width $27.7 \mathrm{~m}$; Subst. bedrock; Shading $0 \%$.

7.** Phormidium corium Gomont ex Gomont, Annales des Sciences Naturelles, Botanique, Série 7 16: 172. 1892.

Plate 4. Figure G.

Mats of filaments, greenish blue; sheath thin, hyaline, homogeneous, sometimes absent; trichomes unconstricted, non granulated in septa; intercalar cells shorter or longer than wide, 2.3-5.9 $\mu \mathrm{m}$ long and 3.8-4.8 $\mu \mathrm{m}$ diam., L/D 0.6-1.3; apical cells conical rounded, 3.4-4.3 $\mu \mathrm{m}$ long and 3.3-4.8 $\mu \mathrm{m}$ diam., RC/D 0.8-1.6; cellular content homogeneous, greenish blue, with few small granules.

Distribution in the Parnaiba River Basin ( $n=1)$ : Site PI-04. BRAZIL, PIAUÍ: $05^{\circ} 1^{\prime} 58.87^{\prime \prime} \mathrm{S}$ and $42^{\circ} 46^{\prime} 41.44$ " W, 43 amsl, 30.V.2017, M.R.Auricchio (UNOP 5779).

Environmental conditions $(n=1)$ : Temp. $28.1^{\circ} \mathrm{C}$; Cond. $301 \mu \mathrm{S} . \mathrm{cm}^{-1}$; pH 6.6; Vel. 0.9 m.s ${ }^{-1}$; Depth $5.4 \mathrm{~cm}$; Width $19.0 \mathrm{~m}$; Subst. bedrock; Shading $31 \%$.

Taxonomic comments: this population was collected in a transitional environment at the stream border, which confers with the description proposed by Komárek \& Anagnostidis (2005), in which P. corium is common in subaerophytic environments.

8.** Phormidium retzii Kützing ex Gomont, Annales des Sciences Naturelles, Botanique, series 7 15: 175. 1892.

Plate 4. Figure F.

Mats of filaments, greenish blue to dark green; sheath thin, hyaline, sometimes absent; trichomes unconstricted and not granulated in septa; intercalar cells shorter or longer than wide, 3.8-8.1 $\mu \mathrm{m}$ long and 5.2-11.4 $\mu \mathrm{m}$ diam., L/D 0.4-1.4; apical cell truncate or rounded, 3.7-11.2 $\mu \mathrm{m}$ long and 5.1-11.4 $\mu \mathrm{m}$ diam., L/D 0.6-1.4; cellular content homogeneous, blue-green, with few small granules.

Distribution in the Parnaiba River Basin ( $n=3)$ : Sites PI-02, PI-15, PI-16. BRAZIL, PIAUÍ: 05 ${ }^{\circ} 19^{\prime} 16.35^{\prime \prime} \mathrm{S}$ and $42^{\circ} 48^{\prime} 29.01^{\prime}$ W, 64 amsl, 29.V.2017, M.R.Auricchio (UNOP 5773); idem, MARANHÃO:

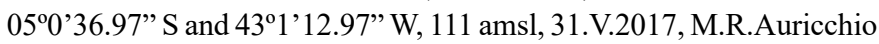

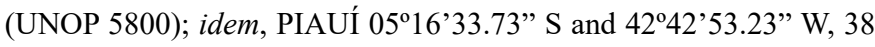
amsl, 01.VI.2017, M.R.Auricchio (UNOP 5807, UNOP 5805).

Environmental conditions $(n=3)$ : Temp. $26.8-27.8^{\circ} \mathrm{C}\left(\overline{\mathrm{x}}=27.1^{\circ} \mathrm{C}\right)$; Cond. 51-321 $\mu{\mathrm{S} . \mathrm{cm}^{-1}}\left(\overline{\mathrm{x}}=156 \mu \mathrm{S} . \mathrm{cm}^{-1}\right)$; $\mathrm{pH}$ 6.4-7.8 $(\overline{\mathrm{x}}=6.9)$; Vel. $0.1-1.2 \mathrm{~m} . \mathrm{s}^{-1}\left(\overline{\mathrm{x}}=0.6 \mathrm{~m} . \mathrm{s}^{-1}\right)$; Depth $5.0-29.0 \mathrm{~cm}(\overline{\mathrm{x}}=19.7 \mathrm{~cm})$; Width 
2.7-14.4 $\mathrm{m}(\overline{\mathrm{x}}=6.7 \mathrm{~m})$; Subst. bedrock and boulders; Shading 32-63\% $(\bar{x}=48 \%)$.

9.** Phormidium tergestinum (Rabenhorst ex Gomont) Anagnostidis \& Komárek, Archiv für Hydrobiologie, Supplement 80: 406. 1988.

Plate 4. Figure I.

Basionym: Oscillatoria tenuis var. tergestina Rabenhorst ex Gomont 1892.

Mats of filaments, dark blue; sheath thin, hyaline, homogeneous, generally absent; trichomes unconstricted, granulated in septa; intercalar cells wider than long, 2.2-4.6 $\mu \mathrm{m}$ long and 4.6-6.3 $\mu \mathrm{m}$ diam., L/D 0.3-0.8; apical cells rounded, 2.5-5.5 $\mu \mathrm{m}$ long and 4.2-5.9 $\mu \mathrm{m}$ diam., L/D 0.5-1.0; cellular content homogeneous, bright greenish blue.

Distribution in the Parnaiba River Basin $(n=2)$ : Sites PI-04, PI-06. BRASIL, PIAUÍ: $05^{\circ} 1$ '58.87' $\mathrm{S}$ and $42^{\circ} 46^{\prime} 41.44^{\prime \prime} \mathrm{W}, 43 \mathrm{amsl}$, 30.V.2017, M.R.Auricchio (UNOP 5778); idem, MARANHÃO: 04 50 '57.66" S and 42 47'46.89' W, 90 amsl, 30.V.2017, M.R.Auricchio (UNOP 5781, UNOP 5782).

Environmental conditions $(n=2)$ : Temp. $28.1-28.5^{\circ} \mathrm{C}\left(\overline{\mathrm{x}}=28.3^{\circ} \mathrm{C}\right)$; Cond. 301-492 $\mu{\mathrm{S} . \mathrm{cm}^{-1}}\left(\overline{\mathrm{x}}=396 \mu \mathrm{S} . \mathrm{cm}^{-1}\right) ; \mathrm{pH}$ 5.9-6.6 $(\overline{\mathrm{x}}=6.2)$; Vel. $0.3-0.9 \mathrm{~m} . \mathrm{s}^{-1}\left(\overline{\mathrm{x}}=0.6 \mathrm{~m} . \mathrm{s}^{-1}\right)$; Depth 5.4-6.3 cm $(\overline{\mathrm{x}}=5.8 \mathrm{~cm})$; Width $4.3-19.0 \mathrm{~m}(\overline{\mathrm{x}}=11.6 \mathrm{~m})$; Subst. bedrock and sand; Shading $31-88 \%$ $(\overline{\mathrm{x}}=59.5 \%)$.

Taxonomic comments: the identification agrees with the description of P. tergestinum by Komárek \& Anagnostidis (2005), however, according to these authors, this concept is broad, being likely to correspond to a set of species.

10.*** Phormidium sp.

Plate 4. Figure $\mathrm{H}$.

Mats of filaments, dark green; sheath thin, hyaline, generally absent, not lamellated; trichomes unconstricted, not granulated in septa; intercalar cells wider than long, 4.4-8.9 $\mu \mathrm{m}$ long and 9.6-11.8 $\mu \mathrm{m}$ diam., L/D 0.40.8 ; apical cell truncate or rounded, 5.9-11.0 $\mu \mathrm{m}$ long and 10.0-11.9 $\mu \mathrm{m}$ diam., L/D 0.6-1.0; cellular content homogeneous, blue-green, with few small granules.

Distribution in the Parnaiba River Basin $(n=2)$ : Sites PI-10, PI-18. BRAZIL, MARANHÃO: 05'3'36.68" S and 42'53'38.29" W, 67 amsl, 31.V.2017, M.R.Auricchio (UNOP 5787); idem, PIAUÍ: 05²2'27.19”' $\mathrm{S}$ and $42^{\circ} 38^{\prime} 40.05^{\prime} \mathrm{W}, 71$ amsl, 01.VI.2017, M.R.Auricchio (UNOP 5818).

Environmental conditions $(n=2)$ : Temp. $27.6-29.4^{\circ} \mathrm{C}\left(\overline{\mathrm{x}}=28.5^{\circ} \mathrm{C}\right)$; Cond. 51-79 $\mu \mathrm{S} . c m^{-1}\left(\overline{\mathrm{x}}=65 \mu \mathrm{S} . \mathrm{cm}^{-1}\right) ; \mathrm{pH}$ 7.0-7.3 ( $\left.\overline{\mathrm{x}}=7.1\right)$; Vel. 0.11-0.8 $\mathrm{m} . \mathrm{s}^{-1}\left(\overline{\mathrm{x}}=0.45 \mathrm{~m} \cdot \mathrm{s}^{-1}\right)$; Depth 11.3-11.7 cm $(\overline{\mathrm{x}}=11.5 \mathrm{~cm})$; Width 5.1-12.1 $\mathrm{m}(\overline{\mathrm{x}}=8.6 \mathrm{~m})$; Subst. bedrock; Shading $62-86 \%(\overline{\mathrm{x}}=74 \%)$.

Taxonomic comments: although the cellular dimensions are within the predicted in the description of $P$. retzii proposed by Komárek \& Anagnostidis (2005), the other characters (e.g. coloration and granulation) present considerable differences with the populations of $P$. retzii identified here. On the other hand, the characteristics of the material do not allow identification in other species. Thus, it is quite likely that this is a new species to be confirmed by future studies including ultrastructural or molecular characters.

\section{DIVISION RHODOPHYTA}

CLASS COMPSOPOGONOPHYCEAE

ORDER COMPSOPOGONALES

1.* Compsopogon caeruleus (Balbis ex C. Agardh) Montagne, Sciences physiques. Botanique. Cryptogamie 1: 154. 1846.

Plate 5. Figure A.

Basionym: Conferva caerulea Balbis ex C.Agardh 1824.

Branched filamentous, dark blue to reddish brown; apical filament uniaxial, uncorticated and branched, older mid and basal filaments corticated, without rhizoidal filaments; axial cells doliform or sub-spherical, 53.5-190.0 $\mu \mathrm{m}$ long and 132.0-335.1 $\mu \mathrm{m}$ diam., L/D 0.4-0.6; cortication formed by regular divisions of the axial cells; external cortical cells generally polygonal, 17.8-27.6 $\mu \mathrm{m}$ long and 12.9-21.5 $\mu \mathrm{m}$ diam., L/D 1.0-1.7.

Distribution in the Parnaiba River Basin $(n=2)$ : Sites PI-02, PI-17. BRAZIL, PIAUÍ: $05^{\circ} 19^{\prime} 16.35^{\prime \prime} \mathrm{S}$ and $42^{\circ} 48^{\prime} 29.01^{\prime \prime} \mathrm{W}, 64 \mathrm{amsl}$, 29.V.2017, M.R.Auricchio (UNOP 5774); idem, 05'21'20.8' S and 4245'20.91" W, 59 amsl, 01.VI.2017, M.R.Auricchio (UNOP 5812). Environmental conditions $(n=2)$ : Temp. $26.8-29.1^{\circ} \mathrm{C}\left(\overline{\mathrm{x}}=27.9^{\circ} \mathrm{C}\right)$; Cond. 316-321 $\mu{\mathrm{S} . \mathrm{cm}^{-1}}^{-1}\left(\overline{\mathrm{x}}=318 \mu \mathrm{S} . \mathrm{cm}^{-1}\right) ; \mathrm{pH}$ 7.8-8.0 ( $\left.\overline{\mathrm{x}}=7.9\right)$; Vel. 0.4-0.5 m.s. ${ }^{-1}\left(\overline{\mathrm{x}}=0.5 \mathrm{~m} . \mathrm{s}^{-1}\right)$; Depth $5.0-20.6 \mathrm{~cm}(\overline{\mathrm{x}}=12.8 \mathrm{~cm})$; Width $2.0-14.4 \mathrm{~m}(\overline{\mathrm{x}}=8.2 \mathrm{~m})$; Subst. bedrock and boulders; Shading $48-63 \%$ $(\overline{\mathrm{x}}=55 \%)$.

\section{CLASS FLORIDEOPHYCEAE}

ORDER BATRACHOSPERMALES

1.* 'Chantransia' macrospora

Plate 5. Figure B.

Uniseriate branched filaments forming tufts. Branches with angles greater than $25^{\circ}$; generally greyish green to reddish brown; cells of the main filaments cylindrical with 25.2-85.0 $\mu \mathrm{m}$ long and 9.2-20.1 $\mu \mathrm{m}$ diam., L/D 2.2-4.7; monosporangia spherical, or in a few cases, obovoidal, 22.5-34.5 $\mu \mathrm{m}$ in diameter.

Distribution in the Parnaiba River Basin $(n=2)$ : Sites PI-15, PI-19. BRAZIL, MARANHÃO: $05^{\circ} 0$ '36.97' S and 43'1'12.97' W, $111 \mathrm{amsl}$, 31.V.2017, M.R.Auricchio (UNOP 5799); idem, PIAUÍ: 05²2'45.37”' S and 42'39'1.548' W, 68 amsl, 01.VI.2017, M.R.Auricchio (UNOP 5819).

Environmental conditions $(n=2)$ : Temp. $26.9-29.4^{\circ} \mathrm{C}\left(\overline{\mathrm{x}}=28.1^{\circ} \mathrm{C}\right)$; Cond. 51-130 $\mu \mathrm{S} . \mathrm{cm}^{-1}\left(\overline{\mathrm{x}}=90 \mu \mathrm{S} . \mathrm{cm}^{-1}\right) ; \mathrm{pH}$ 6.6; Vel. 0.7-1.2 m.s ${ }^{-1}$ $\left(\overline{\mathrm{x}}=0.9 \mathrm{~m} . \mathrm{s}^{-1}\right)$; Depth 17.6-25.2 cm $(\overline{\mathrm{x}}=21.4 \mathrm{~cm})$; Width $2.1-3.0 \mathrm{~m}$ $(\overline{\mathrm{x}}=2.5 \mathrm{~m})$; Subst. boulders; Shading 32-48\% $(\overline{\mathrm{x}}=40 \%)$.

\section{2.* 'Chantransia' pygmaea}

Plate 5. Figure C.

Uniseriate branched filaments forming tufts. Branches with angles lower than $25^{\circ}$; generally greyish green to reddish brown; cells of the main filaments cylindrical with 24.8-48.4 $\mu \mathrm{m}$ long and 5.2-10.9 $\mu \mathrm{m}$ diam., L/D 3.5-5.7; monosporangia obovoidal to spherical, 11.7-25.8 $\mu \mathrm{m}$ in diameter.

Distribution in the Parnaiba River Basin $(n=2)$ : Sites PI-02, PI-19. BRAZIL, PIAUÍ: $05^{\circ} 19{ }^{\prime} 16.35^{\prime \prime} \mathrm{S}$ and 42 48'29.01" W, 64 amsl, 29.V.2017, M.R.Auricchio (UNOP 5771); idem, 05'22'45.37' S and 42³9'1.548” W, 68 amsl, 01.VI.2017, M.R.Auricchio (UNOP 5819). 
Stream macroalgal flora from Parnaíba
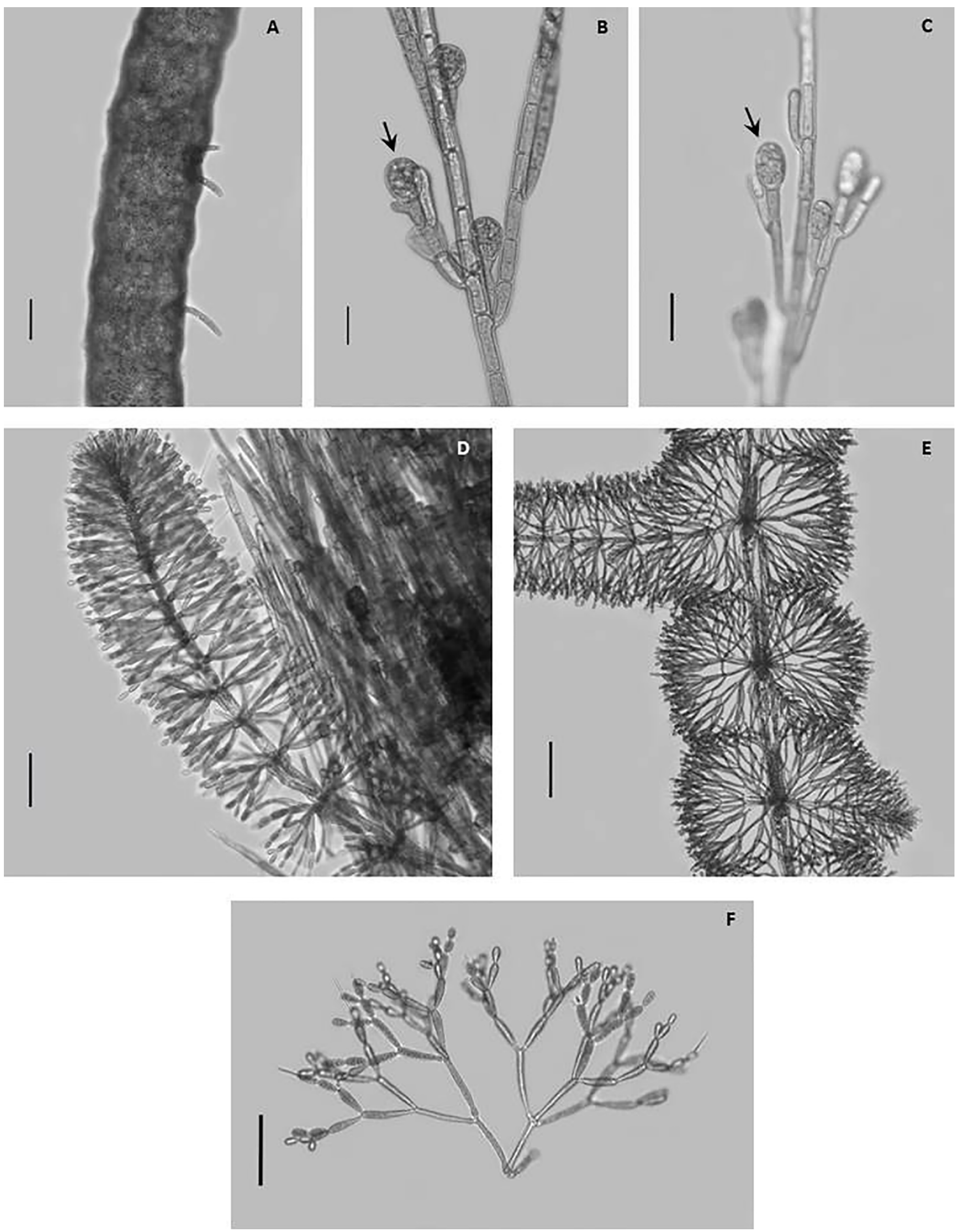

Plate 5. A; Compsopogon caeruleus; B. 'Chantransia' macrospora with monosporangia (arrow); C. 'Chantransia' pygmaea with monosporangia (arrow); D-F. Batrachospermum cf. macrosporum: D. B. cf. macrosporum associated with 'Chantransia' macrospora; E. General view of the whorls; F. Primary fascicle. Scale bars $=100 \mu \mathrm{m}$ (Figures A, D, F); $20 \mu \mathrm{m}$ (Figures B-C); $200 \mu \mathrm{m}$ (Figure E). 
Environmental conditions $(n=2)$ : Temp. $26.8-29.4^{\circ} \mathrm{C}\left(\overline{\mathrm{x}}=28.1^{\circ} \mathrm{C}\right)$; Cond. 130-321 $\mu{\mathrm{S} . \mathrm{cm}^{-1}}^{-1}\left(\overline{\mathrm{x}}=225 \mu \mathrm{S} . \mathrm{cm}^{-1}\right)$; $\mathrm{pH}$ 6.6-7.8 $(\overline{\mathrm{x}}=7.2)$; Vel. $0.5-0.8 \mathrm{~m} \cdot \mathrm{s}^{-1}\left(\overline{\mathrm{x}}=0.6 \mathrm{~m} \cdot \mathrm{s}^{-1}\right)$; Depth $5.0-17.6 \mathrm{~cm}(\overline{\mathrm{x}}=11.3 \mathrm{~cm})$; Width 2.1-14.4 m $(\overline{\mathrm{x}}=8.2 \mathrm{~m})$; Subst. bedrock; Shading 48-63\% $(\overline{\mathrm{x}}=55 \%)$.

3.* Batrachospermum cf. macrosporum Montagne, Annales des Sciences Naturelles, Botanique, Troisième série 14: 293. 1850.

Plate 5. Figure D-F.

Multiseriate filaments moderately mucilaginous, irregularly and abundantly branched; straight apex, whorls well developed, lax, spherical or doliform, generally contiguous, 577.6-1642.1 $\mu \mathrm{m}$ diam.; internodes with 234.1-715.6 $\mu \mathrm{m}$ long and 37.1-71.8 $\mu \mathrm{m}$ diam., L/D 5.9-10.1; primary fascicles with 5-7 cells, 2-4 di- or trichotomously branched; proximal cells ellipsoidal or cylindrical, 55.4-97.7 $\mu \mathrm{m}$ long and 6.9-12.5 $\mu \mathrm{m}$ diam., L/D 4.4-11.8; distal cells ellipsoidal or obovoidal, 16.7-35.5 $\mu \mathrm{m}$ long and 7.9-15.5 $\mu \mathrm{m}$ diam., L/D 1.2-3.9; carposporophytes and carpogonia non observed.

Distribution in the Parnaiba River Basin $(n=1)$ : Site PI-15. BRAZIL, MARANHÃO: 050'36.97' S and 43'1'12.97' W, 111 amsl, 31.V.2017, M.R.Auricchio (UNOP 5802).

Environmental conditions $(n=1)$ : Temp. $26.9^{\circ} \mathrm{C}$; Cond. $51 \mu \mathrm{S} . \mathrm{cm}^{-1}$; pH 6.6; Vel. 1.2 m.s ; $^{-1}$ Depth $25.2 \mathrm{~cm}$; Width $3.0 \mathrm{~m}$; Subst. boulders; Shading $32 \%$.

Taxonomic comments: the population sampled did not have carposporophytes and carpogonia, which would make identification impossible. However, based on vegetative morphology (particularly shaped and size of primary fascicles) and specimens were closely associated with 'Chantransia' macrospora, which is the sporophyte phase of B. macrosporum (Necchi Júnior \& Zucchi 1997). Thus, we indicate this species as conferatum until a more precise identification.

\section{4.* Batrachospermum sp.}

Plate 6. Figure A-C.

Multiseriate filaments moderately mucilaginous, irregularly and abundantly branched; straight apex, whorls well developed, dense or lax, obconical or doliform, generally contiguous, 202.4-350.5 $\mu \mathrm{m}$ diam.; internodes 110.4-531.6 $\mu \mathrm{m}$ long and 32.0-72.0 $\mu \mathrm{m}$ diam., L/D 3.4-10.0; primary fascicles with 6-9 cells, 2-3 di- or trichotomously branched; proximal cells cylindrical, 16.5-26.8 $\mu \mathrm{m}$ long and 4.7-7.2 $\mu \mathrm{m}$ diam., L/D 3.2-5.5; distal cells elliptical or obovoidal, 8.6-11.7 $\mu \mathrm{m}$ long and 5.2-7.3 $\mu \mathrm{m}$ diam., L/D 1.2-1.8; numerous secondary fascicles along the internode length, usually reach the length of the primary fascicles; symmetrical carpogonia with 28.7-47.6 $\mu \mathrm{m}$ long and 6.1-9.0 $\mu \mathrm{m}$ diam., L/D 3.1-7.8; carposporophytes not observed.

Distribution in the Parnaiba River Basin ( $n=1)$ : Site PI-15. BRAZIL, MARANHÃO: 05ㅇ'36.97' S and 43'1'12.97' W, 111 amsl, 31.V.2017, M.R.Auricchio (UNOP 5802-1).

Environmental conditions $(n=1)$ : Temp. $26.9^{\circ} \mathrm{C}$; Cond. $51 \mu \mathrm{S} . \mathrm{cm}^{-1}$; pH 6.6; Vel. 1.2 m.s $\mathrm{s}^{-1}$; Depth $25.2 \mathrm{~cm}$; Width $3 \mathrm{~m}$; Subst. boulders; Shading $32 \%$.

Taxonomic comments: no carposporophytes were observed in the population, making it impossible to identify the material at a specific level.

5.** Kumanoa abilii Necchi (Reis) Necchi Júnior \& Vis, Phycologia 49(1): 101. 2010.
Plate 6. Figure D-G.

Basionym: Batrachospermum abilii M.P. dos Reis 1965.

Free multiseriate filaments with moderate mucilage, irregularly and abundantly branched; straight apex, whorls well developed, dense or lax, obconical or doliform, generally contiguous, with 199.3-451.9 $\mu \mathrm{m}$ diam. internodes with 56.7-459.5 $\mu \mathrm{m}$ long and 15.9-60.5 $\mu \mathrm{m}$ diam., L/D 2.3-10.5; primary fascicles with 5-11 cells, 2-4 di or trichotomously branched; proximal cylindrical cells with 16.1-30.1 $\mu \mathrm{m}$ long, 4.1-9.0 $\mu \mathrm{m}$ diam., L/D 1.8-6.1; distal cells elliptical, obovoidal or spherical with 4.8-13.2 $\mu \mathrm{m}$ long and 4.3-7.8 $\mu \mathrm{m}$ diam., L/D 0.9-2.2; numerous secondary fascicles along the internode length, usually reach the length of the primary fascicles; curved carpogonial branches, differentiated from fascicles, on pericentral cells; asymmetrical carpogonia, 25.7-44.1 $\mu \mathrm{m}$ long, 4.0-8.6 $\mu \mathrm{m}$ diam., L/D 3.7-7.8; carposporophytes sessile, 1 per whorl, dense, semi-spherical, 112.8-194.1 $\mu \mathrm{m}$ diam; compact mass of gonimoblast filaments, carposporangia formed at apices with 9.5-10.2 $\mu \mathrm{m}$ diam.

Distribution in the Parnaiba River Basin ( $n=1)$ : Site PI-18. BRAZIL, PIAUÍ: $05^{\circ} 22^{\prime} 27.19^{\prime \prime} \mathrm{S}$ and $42^{\circ} 38^{\prime} 40.05^{\prime} \mathrm{W}, 71 \mathrm{amsl}$, 01.VI.2017, M.R.Auricchio (UNOP 5817).

Environmental conditions $(n=1)$ : Temp. $29.4^{\circ} \mathrm{C}$; Cond. $79 \mu \mathrm{S} . \mathrm{cm}^{-1} ; \mathrm{pH}$ 7.0; Vel. 0.1 m.s. ${ }^{-1}$; Depth $11.3 \mathrm{~cm}$; Width $5.1 \mathrm{~m}$; Subst. rock, boulders, gravel; Shading $62 \%$.

Taxonomic comments: the carposporophytes measures were slightly smaller when compared to what was described by Kumano (2002) (150.0-300.0 $\mu \mathrm{m})$ to Batrachospermum abilii, however, the morphological characteristics confer with what was described for the species.

\section{Discussion}

The total number of taxa found in the present study (38) can be considered high when compared to studies carried out in other Brazilian regions that used similar methodology, namely: i) 13 taxa (Pereira \& Branco 2010) and 21 taxa (Almeida et al. 2011) in the northwest of the São Paulo State; ii) 23 taxa (Branco et al. 2008) and 34 taxa (Krupek et al. 2008), in the central-southern region of the Paraná State; iii) 24 taxa (Branco et al. 2009) in the mid-western region of the Paraná State, and; iv) 19 taxa (Peres et al. 2008) reported to the eastern region of Paraná State. Likewise, when considering the relative richness, found 1.81 taxa per sampling point, which is also higher when compared to what was reported in those other regions (taxa per sampling point and respective studies: 1.09 in Branco et al. 2009, 1.23 in Almeida et al. 2011, 1.35 in Peres et al. 2008, 1.78 in Krupek et al. 2008, 2.09 in Branco et al. 2008). Studies in tropical streams have shown that biomes without a dense canopy cover can sustain richer macroalgae communities (Necchi Júnior et al. 2003, 2008). Apparently, a larger radiation area in the drainage basin (which is typical in the Caatinga and Cerrado biomes, here sampled) may provide support for a bigger regional species pool that enables greater local richness (as demonstrated for green algae in Peres et al. al. 2017).

In terms of the algal groups that are commonly found in streams, there were predominant species of green algae (Chlorophyta plus Charophyta) (13 taxa, 34.2\%) followed by Cyanophyta (10 taxa, 26.3\%). These data corroborate the pattern observed by Sheath \& Cole (1992, $35 \%$ and $24 \%$, green algae and cyanobacteria, respectively), Vis et al. 
Stream macroalgal flora from Parnaíba
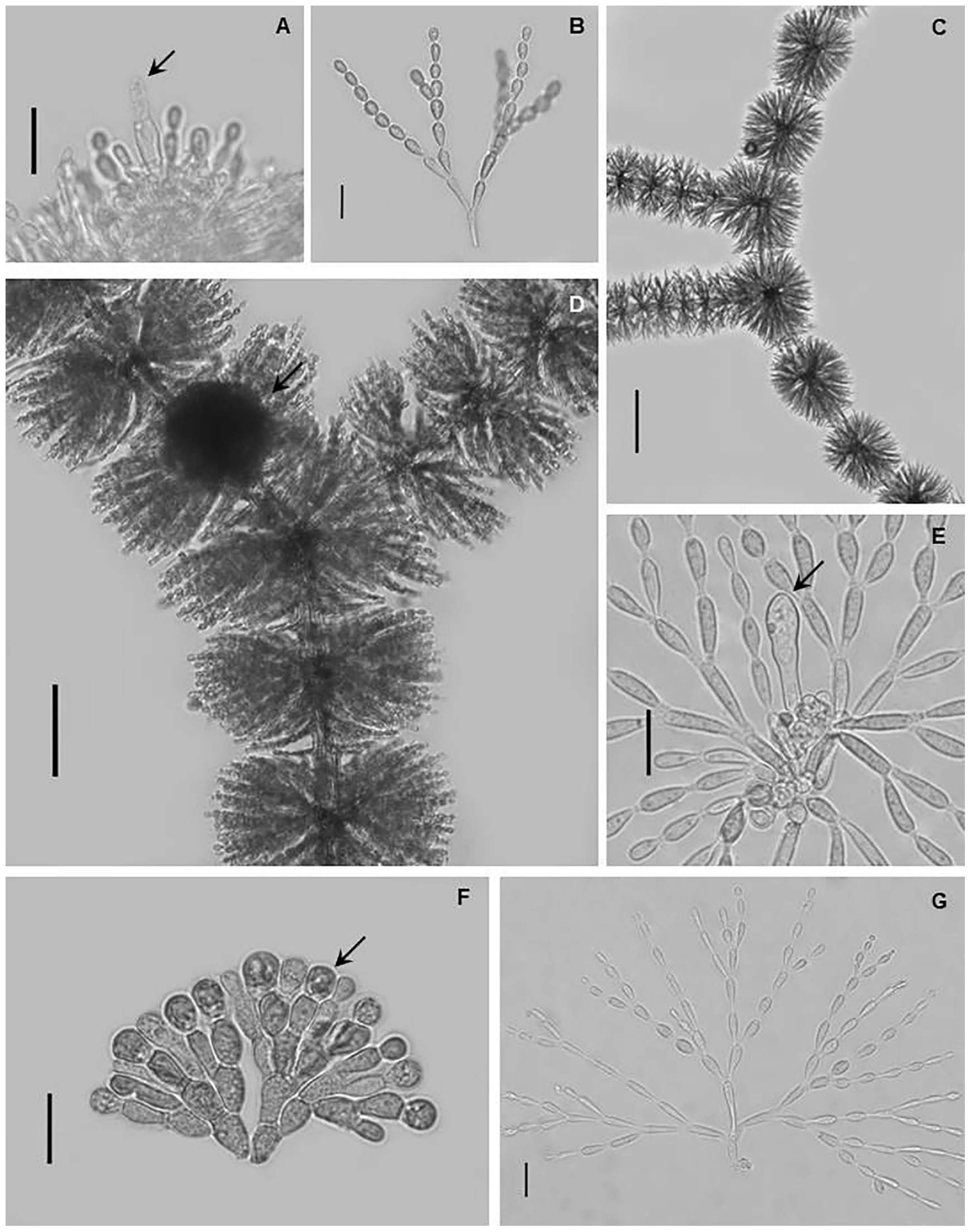

Plate 6. A-C. Batrachospermum sp.: A. Carpogonia (arrow); B. Primary fascicle; C. General view of the whorls. D-G. Kumanoa abilii: D. General view of the whorls with a carposporofite (arrow); E. Carpogonia (arrow); F. Gonimoblast filaments with carposporangia at apices (arrow); G. Primary fascicle. Scale bars $=20 \mu \mathrm{m}$ (figs. A-B, E-G);100 $\mu \mathrm{m}$ (fig. D); $200 \mu \mathrm{m}$ (fig. C). 
(1994, 47\% and 32\%), Branco et al. (2008, 52.2\% and 26.1\%), Branco et al. (2009, 54\% and 21\%), Almeida et al. (2011, 43\% and 38\%) and Krupek \& Branco (2014, 58.4\% and 25\%). Most surveys cited above include disturbed regions or open riparian vegetation. It appears that this predominance of green algae species in these regions occurs due to the better adaptation of the group to high light intensity regimes due to characteristics of its photosynthetic complex (Branco et al. 2017). Although Bacillariophyta was not the richest group, in this study a bigger number of diatom species was found than conventionally for stream macroalgae studies (e.g. Necchi et al. 2000, Branco et al. 2008, 2009). Even though unicellular, diatoms can form monospecific macroscopic colonies (Biggs \& Kilroy 2000, as shown in the Plate 1, Figure A in this study) that can dominate the streambed - hence macroalga, sensu Sheath \& Cole (1992).

The results of this work make very clear the importance of floristic surveys to the reduction of the algae biodiversity conservation shortfalls, specially the Wallacean Shortfall (Whittaker et al. 2005). Of the 38 species recorded by this taxonomic survey, 37 are new records for the Parnaíba River Basin where only Cladophora glomerata was already registered for the basin. Following the same pattern, 23 species are new records for the Brazilian Northeast region (60.5\% of the total) and Microcoleus lacustris represented the first record for the Brazilian territory. Despite the fact that it remains the Brazilian watershed with the lowest number of algal species documented, this work contributed to the increase of almost five times the number of species sampled in the Parnaíba River Basin (from only 10 to 47 species, Flora do Brasil 2020 under construction 2018). These data reinforce that the differences presented in the algal diversity in the Brazilian regions are much more related to the sampling effort than to environmental differences. In addition, this study illustrates not only the group's lack of information in the region but also shows the importance of this type of study as a tool for expanding knowledge about biodiversity and its conservation.

\section{Acknowledgements}

We are grateful to PRPPG/UNILA for support (Edital 109/2017) and field work help provided by Paulo Auricchio, Pedro Henrique Soares Melo, Caio Frederico Saraiva da Silveira, Gabriel Saraiva Lima e Silva, Yasnailson Euflávio de Souza and Cláudia Renata Madella-Auricchio.

\section{Author Contributions}

Marina Ramos Auricchio: Contributed in the concept and design of the study, data collection and analysis, and manuscript preparation.

Richard Wilander Lambrecht: Contributed in the data analysis, taxonomic confirmation and manuscript preparation.

Cleto Kaveski Peres: Contributed in the data analysis, taxonomic confirmation and manuscript preparation.

\section{Conflicts of interest}

The authors declare that they have no conflict of interest related to the publication of this manuscript.

\section{References}

ALMEIDA, F.V., NECCHI JÚNIOR, O. \& BRANCO, L.H.Z. 2011. Flora de comunidades de macroalgas lóticas de fragmentos florestais remanescentes da região noroeste do Estado de São Paulo, Brasil. Hoehnea 38(4):553-568.

AGÊNCIA NACIONAL DE ÁGUAS (BRASIL). 2015. Conjuntura dos recursos hídricos no Brasil: regiões hidrográficas brasileiras - Edição Especial. Brasília: ANA.

AZEVEDO, M.T.P., NOGUEIRA, N.M.C. \& SANT'ANNA, C.L. 1996. Criptógamos do Parque Estadual das Fontes do Ipiranga, São Paulo, SP. Algas, 8: Cyanophyceae. Hoehnea 23:1-38.

BICUDO, C.E. \& MENEZES, M. 2006. Gêneros de algas de águas continentais do Brasil: chave para identificação e descrição. RiMa, São Carlos.

BIGGS, B.J.F. \& KILROY, C. 2000. Stream periphyton monitoring manual. Published by NIWA for Ministry for the Environment.

BINI, L.M., DINIZ-FILHO, J.A.F., RANGEL, T.F.L.V.B., BASTOS, R.P, \& PINTO, M.P. 2006. Challenging Wallacean and Linnean shortfalls: knowledge gradients and conservation planning in a biodiversity hotspot. Divers. Distrib. 12(5):475-482.

BRANCO, C.C.Z., PERES, C.K., KRUPEK, R.A. \& BERTUSSO, F.R. 2009 Stream macroalgae from the Midwestern region of Paraná State, southern Brazil. Biota Neotrop. 9(2):227-235.

BRANCO, C.C.Z., EMED, R.G., BRANCO, L.H.Z. \& NECCHI JUNIOR, O. 2008. Macroalgas de riachos da Floresta Nacional de Irati, região centro-sul do Estado do Paraná, Sul do Brasil. Acta Bot. Bras. 22(2):437-451.

BRANCO, C.C.Z., RIOLFI, T.A., CRULHAS, B.P., TONETTO, A.F., BAUTISTA, A.I.N. \& NECCHI JÚNIOR, O. 2017. Tropical lotic primary producers: Who has the most efficient photosynthesis in low-order stream ecosystems? Freshw. Biol. 62:1623-1636.

BRANCO, C.C.Z., RIOLFI, T.A., PERES, C.K. \& NECCHI JUNIOR, O. 2011. Rhodophyta de ambientes lóticos de Unidades de Conservação da região Sul do Brasil. Biota Neotrop. 11(3): 365-375.

BRANCO, C.C.Z. \& NECCHI JÚNIOR, O. 1996. Distribution of stream macroalgae in the eastern Atlantic Rainforest of São Paulo State, southeastern Brazil. Hydrobiologia 333(3):139-150.

BRANCO, C.C.Z.; NECCHI JÚNIOR, O. \& BRANCO, L.H.Z. 2002. Taxonomy and ecological distribution of Chaetophoraceae (Chaetophorales, Chlorophyta) in lotic ecosystems from São Paulo State, southeastern Brazil. Algol. Stud. 106:43-75.

BROWN, J. H. \& LOMOLINO, M. V. 1998. Biogeography. Sinauer Associates, USA.

COSTA, L.F., WETZEL, C.E., LANGE-BERTALOT, H., ECTOR, L. \& BICUDO, D.C. 2017. Taxonomy and ecology of Eunotia species (Bacillariophyta) in southeastern Brazilian reservoirs. Bibl Diatomol. 64: 302.

DESIKACHARY, T.V. 1959. Cyanophyta. Indian Council of Agricultural Research, New Delhi.

FLORA DO BRASIL 2020 UNDER CONSTRUCTION. 2018. (http:// floradobrasil.jbrj.gov.br). Último acesso em: 29/06/2018.

GORDON, N.D., McMAHON, T.A. \& FINLAYSON, B.L. 1992. Stream Hydrology: an introduction for ecologists. Chichester, John Wiley \& Sons.

GUIRY, M.D. \& GUIRY, G.M. 2018. AlgaeBase. World-wide electronic publication, National University of Ireland, Galway. Disponível em: $<$ http://www.algaebase.org> Acessado em: jun. 2018

KOMÁREK, J. \& ANAGNOSTIDIS, K. 2005. Cyanoprokaryota 2.Teil: Oscillatoriales. Elsevier $\mathrm{GmbH}$, Munchen.

KRUPEK, R.A., BRANCO, C.C.Z. \& PERES, C.K. 2008. Levantamento florístico das comunidades de macroalgas da bacia do rio das Pedras, região centro-sul do Estado do Paraná, Sul do Brasil. Hoehnea 35(2):189-208.

KRUPEK, R.A. \& BRANCO, C.C.Z. 2014. Estrutura das comunidades de macroalgas da bacia de drenagem do rio Cascavel, Estado do Paraná, Brasil. Hoehnea 41(1):41-50. 
KUMANO, S. 2002. Freshwater red algae of the world. Biopress Ltd, Bristol. LOMOLINO, M.V. 2004. Conservation biogeography. In: LOMOLINO M.V. \& HEANEY L.R. (Eds.) Frontiers of Biogeography: new directions in the geography of nature. Sinauer Associates, Sunderland, Massachusetts.

MACE, G.M. 2004. The role of taxonomy in species conservation. Philos. T. R. Soc. B 359(1444): 711-719.

MENEZES, M., BICUDO, C.E.M., MOURA, W.N., ALVES, A.M., SANTOS, A.A., PEDRINI, A.G., ARAÚJO, A., TUCCI, A., FAJAR, A., MALONE, C., KANO, C.H., SANT'ANNA, C., BRANCO, C.C.Z., ODEBRECHT, C., PERES, C.K., NEUHAUS, E.B., ESKINAZI-LEÇA, E., AQUINO, E., NAUER, F., SANTOS, G.N., AMADO FILHO, G.M., LYRA, G.M., BORGES, G.C.P., COSTA, I.O., NOGUEIRA, I.S., OLIVEIRA, I.B., PAULA, J.C., NUNES, J.M.C., LIMA, J.C., SANTOS, K.R.S., FERREIRA, L.C., GESTINARI, L.M.S., CARDOSO, L.S., FIGUEIREDO, M.A.O., SILVA, M.H., BARRETO, M.B.B.B., HENRIQUES, M.C.O., CUNHA, M.G.G.S., BANDEIRA-PEDROSA, M.E., OLIVEIRA-CARVALHO, M.F., SZÉCHY, M.T.M., AZEVEDO, M.T.P., OLIVEIRA, M.C., CABEZUDO, M.M., SANTIAGO, M.F., BERGESH, M., FUJII, M.T., BUENO, N.C., NECCHI JUNIOR, O., JESUS, P.B., BAHIA, R.G., KHADER, S., ALVES-DA-SILVA, S.M., GUIMARÃES, S.M.P.B., PEREIRA, S.M.B., CAIRES, T.A., MEURER, T., CASSANO, V., WERNER, V.R., GAMA JÚNIOR, W.A. \& SILVA, W.J. 2015. Update of the Brazilian floristic list of Algae and Cyanobacteria. Rodriguésia 66(4):1047-1062.

METZELTIN, D. \& LANGE-BERTALOT, H. 1998. Tropical Diatoms of South America I: About 700 predominantly rarely known or new taxa representative of the neotropical flora. In: LANGE-BERTALOT, H. Iconographia Diatomologica. Vol. 5. Diversity-Taxonomy-Geobotany. Koeltz Scientific Books. Alemanha: Königstein.

MMA: MINISTÉRIO DO MEIO AMBIENTE. 2006. Caderno da Região Hidrográfica do Parnaíba. MMA, Brasília.

NECCHI JÚNIOR, O. 1990. Revision of the genus Batrachospermum Roth (Rhodophyta, Batrachospermales) in Brasil. J. Cramer, Berlin.

NECCHI JÚNIOR, O. \& ZUCCHI, M.R. 1997. Audouinella macrospora (Acrochaetiaceae, Rhodophyta) is the 'Chantransia' stage of Batrachospermum (Batrachospermaceae). Phycologia 36(3):220-224.

NECCHI JÚNIOR, O.; BRANCO, C.C.Z. \& BRANCO, L.H.Z.. 2000. Distribution of stream macroalgae in São Paulo State, southeastern Brazil. Algol. Stud. Stuttgart, 97:43-57.

NECCHI JÚNIOR, O., BRANCO, C.C.Z. \& SPEZAMIGLIO, D. 2008. Distribuição ecológica de comunidades de macroalgas de ambientes lóticos do Parque Nacional de Itatiaia (MG, RJ), Brasil. Braz. J. Bot. 31:135-145.

NECCHI Jr., O., BRANCO, L.H.Z. \& BRANCO, C.C.Z. 2003. Ecological distribution of stream macroalgal communities from a drainage basin in the Serra da Canastra National Park, Minas Gerais, Southeastern Brazil. Braz. J. Biol. 63:1-12.
NECCHI JÚNIOR, O., SPEZAMIGLIO, D.N., BRANCO, C.C.Z. \& BRANCO, L.H.Z. 2001. Estudo taxonômico e distribuição ecológica do gênero Vaucheria (Xanthophyta, Vaucheriaceae) em ecossistemas lóticos do Estado de São Paulo, Brasil. Hoehnea. 28(3):231-242.

NECCHI JÚNIOR, O., PASCOALOTO, D., BRANCO, C.C.Z. \& BRANCO, L.H.Z. 1997. Stream macroalgal flora from the northwest region of São Paulo State, Southeastern Brazil. Algol. Stud. 84:91-112.

NECCHI JÚNIOR, O., DIP, M.R. \& GOES, R.M. 1991. Macroalgae of a stream in southeastern Brazil: composition, seasonal variation and relation to physical and chemical variables. Hydrobiologia 213(3):241-250.

NOVIS, P. M. 2004. A taxonomic survey of Microspora (Chlorophyceae, Chlorophyta) in New Zealand. New Zeal. J. Bot. 42:153-165.

PEREIRA, J.L. \& BRANCO, L.H. 2010. Macroalgas em nascentes e arredores de riachos na região noroeste do Estado de São Paulo. Hoehnea. 37(3):435-444.

PERES, C.K. \& BRANCO, C.C.Z. 2014. Algas verdes macroscópicas de ambientes lóticos do Sul do Brasil: taxonomia e aspectos ecológicos. Novas Edições Acadêmicas, SIA OmniScriptum Publishing, Letônia, 2014.

PERES, C.K., TONETTO, A.F., GAREY, M.V. \& BRANCO, C.C.Z. 2017. Canopy cover as the key factor for occurrence and species richness of subtropical stream green algae (Chlorophyta). Aquat. Bot. 137:24-29.

PERES, C.K., BRANCO, C.C.Z. \& KRUPEK, R.A. 2008. Macroalgas de riachos da Serra da Prata, leste do Estado do Paraná, sul do Brasil. Acta Bot. Bras. 22(2):333-344.

PYKE, G.H. \& EHRLICH, P.R. 2010. Biological collections and ecological/ environmental research: a review, some observations and a look to the future. Biol. Rev. Camb. Philos. 85(2):247-266.

SENNA, P.A. \& FERREIRA, L.V. 1987. Nostocophyceae (Cyanophyceae) da Fazenda Água Limpa, Distrito Federal, Brasil, 2: Famílias Nostocaceae, Scytonemaceae e Stigonemataceae. Rickia 14: 7-19.

SHEATH, R.G. \& COLE, K.M. 1992. Biogeography of stream macroalgae in North America. J. Phycol. 28(4):448-460.

SKINNER, S. \& ENTWISLE, T.J. 2001. Non-marine algae of Australia: 2. Some conspicuous tuft-forming Cyanobacteria. Telopea 9(3):685-712.

VIS, M.L., SHEATH, R.G., HAMBROOK, J.A. \& COLE, K.M. 1994. Stream macroalgae of the Hawaiian islands: a preliminary study. Pac. Sci. 48(2):175-187.

WHITTAKER, R., ARAÚJO, M.B., JEPSON, P., LADLE, R.J., WATSON, J.E.M. \& WILLIS, K.J. 2005. Conservation biogeography: assessment and prospect. Divers. Distrib. 11(1):3-23. 